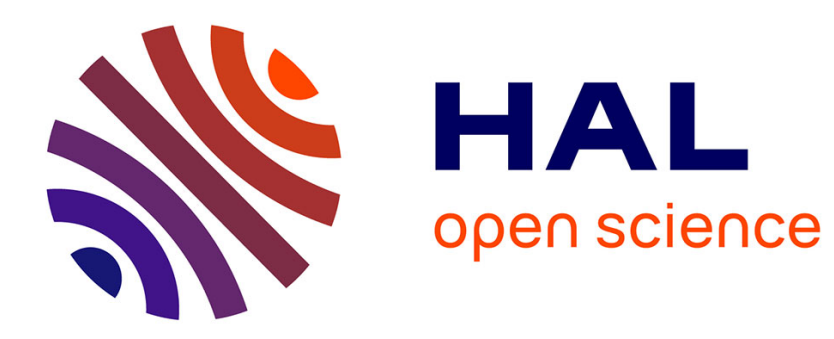

\title{
The geometry of global production and factor price equalisation
}

Ivar Ekeland, Roger Guesnerie

\section{To cite this version:}

Ivar Ekeland, Roger Guesnerie. The geometry of global production and factor price equalisation. 2006. halshs-00589105

\section{HAL Id: halshs-00589105 \\ https://shs.hal.science/halshs-00589105}

Preprint submitted on 27 Apr 2011

HAL is a multi-disciplinary open access archive for the deposit and dissemination of scientific research documents, whether they are published or not. The documents may come from teaching and research institutions in France or abroad, or from public or private research centers.
L'archive ouverte pluridisciplinaire HAL, est destinée au dépôt et à la diffusion de documents scientifiques de niveau recherche, publiés ou non, émanant des établissements d'enseignement et de recherche français ou étrangers, des laboratoires publics ou privés. 


\section{Paris-Jourdan ScIences Economiques \\ 48, BD JOURDAN - E.N.S. - 75014 PARIS \\ TEL. : 33(0) 143136300 - FAX : 33 (0) 143136310 \\ www.pse.ens.fr}

WORKING PAPER N² 2006 - 50

The geometry of global production

and factor price equalisation

Ivar Ekeland

Roger Guesnerie

JEL Codes: D24, D51, D57, D58, F11, E11

Keywords: Constant returns to scale, global production set, non-substitution, factor price equalisation, factor values 


\title{
The geometry of Global Production and Factor Price Equalisation.
}

\author{
EKELAND IVAR \\ Canada Research Chair in Mathematical Economics, UBC \\ Guesnerie Roger \\ Collège de France and Paris School of Economics.
}

Revised December 29, 2006*

\begin{abstract}
We consider a production economy where commodities are partitioned into irreproducible factors and reproducible goods, and the production technologies have constant returns to scale. We examine the geometry of the efficient frontier of the global production set and derive theorems of nonsubstitution type. We complement the geometric viewpoint by an algebraic characterization of the efficient frontier, that put emphasis on the "factors values" of goods. We analyse the connections between the prices of goods and the prices of factors. In particular, we show that if the number of goods is at least twice as large as the number of factors, then, generically, the prices of goods uniquely determine the prices of factors.
\end{abstract}

\section{Résumé :}

Nous considérons le secteur productif d'une économie où les biens sont partitionnés en facteurs non reproductibles et biens productibles et les techniques de production ont des rendements constants. Nous examinons la géométrie de la frontière efficace de l'ensemble de production global et dérivons des théorèmes de type théorème de non-substitution. Le point de vue géométrique est complété par une caractérisation algébrique de la frontière efficace, qui met l'accent sur les "valeurs factorielles" des biens. Nous approfondissons, dans ce contexte, les relations entre prix des biens et prix des facteurs et démontrons, que si le nombre de biens est au moins deux fois plus grand que le nombre de facteurs, "génériquement", le prix des biens détermine univoquement le prix des facteurs.

${ }^{*}$ We are grateful to M. Bégorre-Bret, C. Bidard, P.A Chiappori and M.Jerison for helpful discussions. We also thanks for their comments participants to the 2001 NBER general equilibrium conference and to seminars at the universities of Chicago, Paris 10, Toulouse, and the Institute for Advanced Studies in Vienna. 


\section{INTRODUCTION}

We consider in this paper, an abstract economy in which commodities can be partitioned into produced goods and non-reproducible factors of production, henceforth referred to simply as 'goods' and 'factors'. Goods are produced from goods and factors. The production sector, on which we focus attention, consists of a collection of technologies with constant returns to scale, and without joint production.

Our investigation aims at improving upon the present understanding of the geometry of the global production set, in particular of the subset of what we call nonspecialized production plans and of its efficient frontier. The analysis is conducted both in the primal setting, the space of goods and factors, where we look at the the global production set and its frontier, and in the dual setting, the space of prices, where we investigate the structure of the set of price systems which support efficient production. The conclusions obtained from the complementary combination of the primal and dual viewpoints allows a geometric and algebraic assesment of the qualitative properties of the efficiency frontier under scrutiny. The concept of factor values of the goods provides an intuitive interpretation of the findings. The analysis emphasizes the role prices of goods and the prices of factors. It leads us to revisit in our setting the problem known in the literature as factor price equalisation.

This paper undoubtedly belongs to the field of production theory. However, this microeconomics topics may be related with a larger set of preocupations which have surfaced in the economic literature over a rather long period.

Indeed, special cases of the abstract model we are considering appear in different research traditions, some of which are old, associated, for example, with the names of Sraffa, Leontieff, and Heckscher-Ohlin. Sraffa and Leontieff, because we focus attention on the 'production of commodities by means of commodities' (1960) in a constant returns to scale setting. Heckscher-Ohlin, because the standard models of international trade based on their work ${ }^{1}$ stress, as we do here ${ }^{2}$, the dichotomy between factors and goods.

But although these traditions have developed outside the Walrasian tradition (and sometimes against it), the concerns of the paper may also be related with the present state of general equilibrium theory. On the consumption side, since the discussion on the Sonnenschein-Mantel-Debreu Theorem ${ }^{3}$ in the eighties, it has been recognized

\footnotetext{
${ }^{1}$ See for example, Dixit-Norman (1981)

${ }^{2}$ Indeed, the production sector under consideration here can be viewed as the production sector of a generalized Heckscher-Ohlin economy, in which the number of goods or factors is not restricted to two and in which goods and factors, not only factors, serve to produce goods

${ }^{3}$ See Shafer-Sonnenschein(1982) and the references therein
} 
that the most abstract theory used in general equilibrium is, somehow, too general. Effort has then been directed towards the development of more focused settings which may be empirically more relevant, for instance in the direction indicated by Hildenbrand (1994). In a similar way, the analysis presented in this paper can be viewed as part of a research program aimed at constructing a theory of production of intermediate scope, lying somewhere between the most general Arrow-Debreu model and the simplified and sometimes simplistic modelling of production adopted in fields of application.

Fields of applications that might potentially benefit from "a theory of production of intermediate generality" include, as we argue in Ekeland-Guesnerie (2007), macroeconomics and growth theory. More obvious examples of possible fruitful connections are for example, computable general equilibrium models and trade theory. Computable general equilibrium models, which are widely used in applied research, generally have constant returns to scale production sectors, often fitting, as special cases, the framework of our model. Also, the present development of international trade has revived the interest for the phenomenon of factor price equalisation ${ }^{4}$. The present production model provides a core building block of elementary trade theory, that would take into account what W.Ethier (1984) called "higher dimensional issues" 5 .

The results of the paper may be presented in five different categories.

- The paper provides a primal description of the geometry of efficient production plans. In particular, Theorem 4 captures, hopefully in a general and synthetical way, the essence of "non-substitution"6: beyond the polar Arrow-Samuelson case, where the efficient boundary is limited by a hyperplane, the frontier of the global production set displays a regular geometry.

\footnotetext{
${ }^{4}$ Economic historians have reassessed the empirical importance of the phenomenon of factor price equalisation(O'Rourke, Taylor and Williamson (1995) and O'Rourke and Williamson (2000)). And one of the hottest empirical debate in the last twenty years has borne on the extent to which factor price equalisation explains the increase of wages differentials in the US: see for example Freeman (1995), for a lively account of empirical aspects of the debate and of its theoretical background. Although the paper does not claim immediate relevance to such debates, it provides some hopefully fresh view on one of its basic perspectives

${ }^{5}$ This is obviously not a new subject subject : see Samuelson (1953), Mc Kenzie, (1955), JonesScheinkman (1977), Neary (1985), for a somewhat limited sample of the most significant earlier contributions.

Note too, that although the "new" theory of international trade stresses new reasons for gains to trade, in addition to the indirect exchange of factors, it does not dismiss the effect of trade on factor prices. On the whole, the interest in the effect which trading goods may have on factor prices seems to have been rekindled rather than weakened by the contemporary development of international trade.

${ }^{6}$ Some of its corollaries are well known, and if the statement itself is likely to have been understood before, it does not seem to have been formulated as such.
} 
- The paper comes back, from Proposition 11, on the global dual description of the production possibilities by stressing the key role of the factor values matrix, both in the formal statements and in their interpretation.

- A key preparation theorem, Theorem 14 is stated. Although it has interest of its own, it is an intermediate technical tool ${ }^{7}$ crucial to the analysis. It has a simple interpretation: the local property according to which, in a constant returns framework, the prices of goods can be locally written as a function of the prices of factors is shown to be global.

- The analysis of the geometry of the global production set stressed for example in Theorem 4 has an algebraic counterpart stressed in Theorem 17, which provides the "equations" of the factor sections of the efficiency frontier. Theorem 22 and its Corollary characterizes a surrogate production sector where goods would be produced only from factors. Proposition 24 is the key tool for comparative statics studies.

- Finally the paper focuses attention on the factor price equalisation problem that has received a lot of earlier attention. It first reassess and/or extend earlier results.

- Corollary 8 restates one of the early findings of Mc Kenzie.

- Corollary 26 is the adaptation to our more general framework of one of the most powerful standard factor price equalisation theorem ${ }^{8}$ obtained in the GaleNikaido tradition, a tradition that assumes that the number of goods equals the number of factors, together with some generalized non-intensity reversal condition $^{9}$.

- The next global univalence results we give, Theorems 27 and 28 are only generic but do not require any non-intensity reversal assumption. Indeed, theorem 27, our main result, here asserts that if the production plan is efficient, and if the number of goods is at least twice the number of factors, then, generically (that is except for certain pathological and non-robust situations), prices of goods uniquely determine prices of factors.

The paper proceeds as follows.

Section 2 presents the model, the objects under scrutiny.

Section 3 analyses global economic efficiency from the primal viewpoint and provides the basic dual findings..

\footnotetext{
${ }^{7}$ It is straigthforward in some special cases, but not in general.

${ }^{8}$ It is due to Mas Colell(1979b)

${ }^{9}$ See Arrow-Hahn (1971), for an account of the related earlier literature.
} 
Section 4 presents the preparation Theorem and combines it with previous findings in order to obtain a full algebraic characterisation of the efficient frontier, and as a consequence some key comparative statics results.

Section 5 establish the new factor price equalisation statements presented above.

A conclusion is offered and a number of derivations appear in three appendices.

\section{Model AND PRELIMINARY ANALYSis.}

2.1. Setting and assumptions. Consider a static production economy with constant returns to scale, in which one distinguishes between produced goods and non-reproducible factors of production (henceforth referred to as 'goods' and 'factors'). There will be $L$ goods and $K$ factors; we denote a bundle of the former by $x=\left(x^{1}, \ldots, x^{L}\right)$, and of the latter by $v=\left(v^{1}, \ldots, v^{K}\right)$. For the sake of simplicity, we focus on the case where $L \geqq K$., although we will indicate the statements that are valid for $L<K$.

The dual variables will be denoted by $\left(p_{1}, \ldots, p_{L}\right)$, (goods prices) and $\left(w_{1}, \ldots, w_{K}\right)$ (factors remunerations), respectively. The bundle $(x, v) \in R^{L+K}$ will be denoted by $y$, and the system $(p, w) \in R^{L+K}$ by $q$. We shall refer to $y$ as a production plan and to $q$ as a remuneration plan.

Factors cannot be produced but are used to produce goods. Goods must be produced, they are also used to produce other goods. Throughout this paper, we will assume that all technologies available for producing goods exhibit constant returns to scale; such technologies typically use as inputs some factors and some other goods. In this paper, we will assume that:

- each technology has a unique output: there is no joint production,

- all factors and all goods are used in production.

In this setting, the production technology of good $\ell$ will be associated with a production function $f_{\ell}: R_{+}^{L-1} \times R_{+}^{K} \longrightarrow R_{+}$, so that $x^{\ell}=f_{\ell}\left(-x^{\prime},-v\right)$ is the quantity of good $\ell$ that will be produced by using efficiently the available technology and the input vector $\left(x^{\prime}, v\right)$, which has negative components.

Given $x \in R^{L}$, we shall denote by $x^{-\ell} \in R^{L-1}$ the goods bundle obtained from $x$ by deleting the $\ell$-th component. Assuming free disposal, the production set $Y_{\ell}$ for good $\ell$ is then given by:

$$
Y_{\ell}=\left\{(x, v) \in R^{K+L} \mid v \in-R_{+}^{K}, x^{-\ell} \in-R_{+}^{L-1}, x^{\ell} \leq f_{\ell}\left(-x^{-\ell},-v\right)\right\}
$$

Our first assumptions subsume standard conditions concerning production functions, concavity, and constant returns to scale 
Condition 1. For every $\ell$, with respect to all variables the function $f_{\ell}$ is concave and positively homogenous of degree one

The next condition means that all goods other than $\ell$ and all factors are required in producing $\ell$ :

Condition 2. For every $\ell$ the function $f_{\ell}$ is positive on $R_{++}^{L-1} \times R_{++}^{K}$ and vanishes on the boundary.

We shall also need some smoothness and nondegeneracy conditions on the production functions.

Condition 3. For every $\ell$ the function $f_{\ell}$ is twice differentiable on $R_{++}^{L-1} \times R_{++}^{K}$, the first derivatives are all strictly positive:

$$
D f_{\ell}: R_{++}^{L-1} \times R_{++}^{K} \rightarrow R_{++}^{L-1} \times R_{++}^{K}
$$

and the matrix of second derivatives, $D^{2} f_{\ell}$, has corank 1 at every point

The condition on the gradient $D f_{\ell}$ means that the production functions are strictly increasing with respect to all inputs. Since $f_{\ell}$ is positively homogeneous of degree one, $D f_{\ell}$ is positively homogeneous of degree zero, so that, by the Euler identity, we have $D^{2} f_{\ell}(z) z=0$ for all $z \in R_{++}^{L-1} \times R_{++}^{K}$. This means that $z$ is in the kernel of $D^{2} f_{\ell}(z)$, which must be at least one-dimensional. Our assumption means that there is nothing else in the kernel, which is then exactly one-dimensional. It is a standard addition to the smoothness assumption.

Note that, strictly speaking, our assumptions rule out from the analysis two of the more popular models of production economies: the Heckscher-Ohlin model and the Leontieff model. In the Heckscher-Ohlin model, there are two goods and two factors, but no intermediate goods are used in production: goods are produced with factors only. In the Leontieff model, there is a single factor, and for each production sector a single technology: these technologies display strict complementarities, which are incompatible with Condition 3.

Naturally, our model covers cases as close as desired to these two special models. Simple intuition then rightly suggests that most of the results we shall derive here will also apply to these models.

We finally introduce Condition 4.

Condition $4: Y$ is closed, and $\left(R_{++}^{L} \times R^{K}\right) \cap Y \neq \emptyset$.

Condition 4 means that, given sufficient amounts of factors, there is some production plan such that every good is produced in positive net quantity. In other words, the economy is not unproductive to the point where, even with large amounts of factors, it would be impossible to have a positive net production of all goods together ${ }^{10}$

Note already that Condition 4 , together with free disposal and constant returns to scale implies : $\left(R_{++}^{L} \times R^{K}\right) \cap Y=R_{++}^{L}$.

\footnotetext{
${ }^{10}$ Indeed, when the production economy reduces to a Leontieff economy, this condition is the standard condition ensuring that the input-output matrix is is labelled "productive".
} 


\subsection{The global production set. .}

Definitions.. The global production set in the economy is:

$$
Y=\sum_{\ell} Y_{\ell}
$$

We call $Y_{E}$, the efficient frontier of the global production set.

Take a production plan $y=(x, v) \in Y$. By definition, it can be written (possibly in many different ways) as:

$$
y=\sum y_{\ell}
$$

with $y_{\ell}=\left(x_{\ell}, v_{\ell}\right) \in Y_{\ell}$.

This paper focuses attention on production plans that involve all existing production sets, i.e in the just given formal definition, such that $y_{\ell} \neq 0$, (and $y_{\ell}$ non negative). The set of such production plans is a subset of $Y$, denoted $Y_{N S}$.

In other words, the Non Specialized global production $\operatorname{set}^{11} Y_{N S} \subset Y$ is the set of all production plans involving gross (not necesssarily net) positive production of every good.

Again, the knowledge of this set follows from the knowledge of its efficiency frontier :

$$
Y_{N S E}=\left\{y \in Y_{N S} \mid Y \cap\left\{y+R_{++}^{L+K}\right\}=y\right\}
$$

Note that Condition 4 implies that the intersection of $Y_{N S}$, and hence of $Y_{N S E}$, with $R_{++}^{L} \times R^{K}$, is non empty : there is at least one efficient production plan involving the activity of all production sets and involving a gross production of every good.

We call $Y_{N S}^{+}=Y_{N S} \cap\left(R_{++}^{L} \times R^{K}\right)$ and $Y_{N S E}^{+}=Y_{N S E} \cap\left(R_{++}^{L} \times R^{K}\right)$

This paper focuses attention on the non-specialized global production set $Y_{N S}$, its efficient frontier $Y_{N S E}$, and its restriction to the positive orthant of goods $Y_{N S E}^{+}$.

There are several alternative ways to describe production possibilities that provide complementary insights.

We will use three of them.

- The first one relies on the study of the sections of $Y_{\ell}$ with $x^{\ell}=1$, i.e to :

$$
Y_{\ell}^{1}=\left\{(x, v) \in R^{K+L} \mid v \in-R_{+}^{K}, x^{-\ell} \in-R_{+}^{L-1}, 1 \leq f_{\ell}\left(-x^{-\ell},-v\right)\right\}
$$

- The second one focuses on the sections of the global production set, which, for given factors endowments, summarize the production possibilities of the economy .

\footnotetext{
${ }^{11}$ This terminology should not be midsleading. In particular, the properties we are investigating serve to study non specialized situations in trade as well as specialized situations..
} 
The geometry of Global Production and Factor Price Equalisation.

Indeed, for $v \in-R_{++}^{K}$, we define $: Y(., v)=\{x \mid(x, v) \in Y\}$ and, with similar notation, $\quad Y_{N S}(., v), Y_{N S E}(., v)$ and $Y_{N S E}^{+}(., v)$.

- The third one consists in considering sections for fixed $x$, rather than fixed $v$. Indeed, the set $Y(x,)=.\left\{v^{\prime} \mid\left(x^{\prime}, v^{\prime}\right) \in Y, x^{\prime} \geq x\right\}$ is the (convex) set of factor endowments that would allow to produce more than the vector $x$ of goods. We may then consider the sets $Y\left(e_{\ell},.\right), 1 \leq \ell \leq L$, where $e_{\ell}^{i}=0$ if $i \neq \ell$ and $e_{\ell}^{\ell}=1$. (they are non-empty, because of $\mathrm{C} 4$ and its consequences). Viewed as sections of a global production set, they define a surrogate economy where goods are produced from factors alone.

We leave to the reader to check :

Proposition 1. 1. The knowledge of $Y$ is equivalent to the knowledge of $Y_{\ell}^{1}, \forall l$.

2. $Y_{N S}$ can be recovered from $Y_{N S}(., v), \forall v /\|v\|=1$, where $\|$.$\| designates,$ for example, the Euclidean norm.

Efficiency.. The standard decentralisation theorem asserts that an efficient global production plan is the sum of profit-maximizing production plans, profit being measured with an appropriate supporting price vector.

Let us then take a point $y=\sum_{\ell} y_{\ell} \neq 0$ on the efficient frontier of $Y$ (without assuming yet that it is non-specialized). There is some non-trivial price vector $q=$ $(p, w)$, which we call a remuneration plan supporting $y$, such that:

$$
\begin{aligned}
q^{\prime} y & \left.=\operatorname{Max} q^{\prime} z \mid z \in Y\right\} \\
q^{\prime} y_{\ell} & =\operatorname{Max}\left\{q^{\prime} z_{l} \mid z_{l} \in Y_{l}\right\}=0, \text { for every } l .
\end{aligned}
$$

Our conditions C1, C2 straigthforwardly imply

$$
q \in R_{+}^{L+K} \text { and } q^{\prime} y=0
$$

and since we have $y_{\ell} \neq 0$ for some $\ell, \mathrm{C} 3$ involves that $q$ is unique (up to a positive constant).

We have previously normalized the production sets $Y_{\ell}$ by setting production equal to one unit of good $\ell$. From now on, remuneration plans will often be scaled by choosing the first good as numeraire, so that $p_{1}=1$.

$$
E_{1}=q=\left\{(p, w) \in R_{++}^{L+K} \mid p_{1}=1\right\}
$$

Our fist insights are summarized in Proposition 1. 


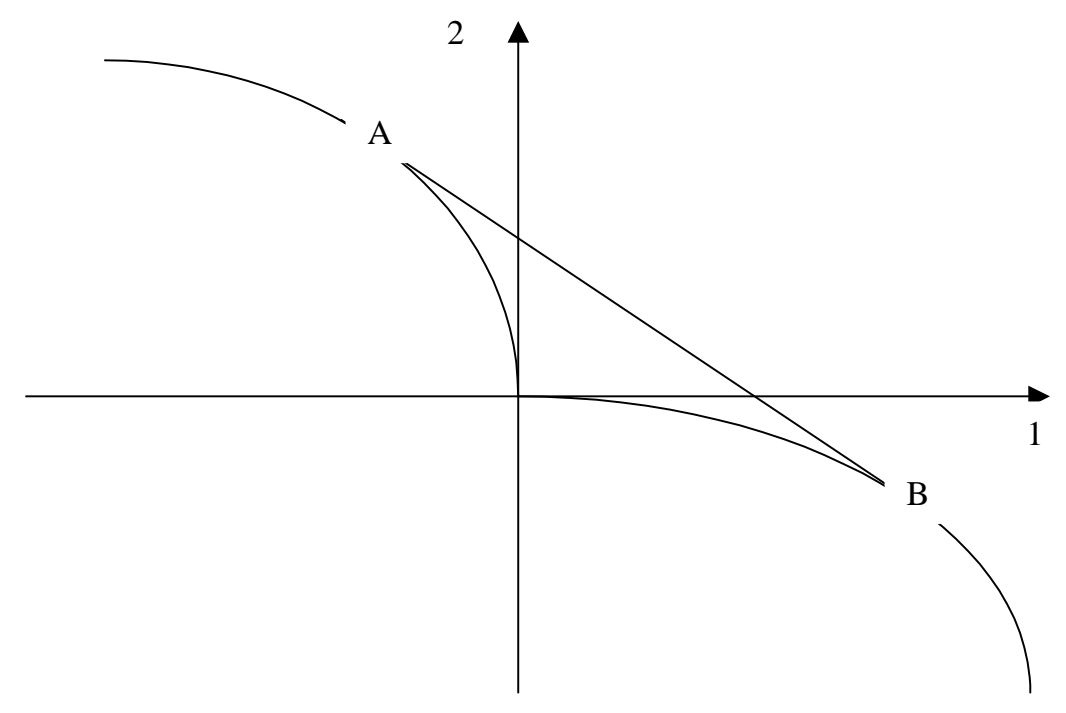

Proposition 2. Let $y$ an efficient NS-production plan and call $\pi(y)$, the unique normalized remuneration plan supporting it. Then :

$-\pi(y) y=0$

- y can be uniquely written as $y=\sum_{\ell} \lambda_{\ell}(y) \gamma_{\ell}(y)$, with $\gamma_{\ell}(y) \in Y_{\ell}^{1}$. and where $\lambda_{\ell}(y)>0$ are the activity levels at which technology $\ell$ has to operate.

$-\pi(y) \gamma_{\ell}(y)=0$, for all $\ell$, or equivalently $\gamma_{\ell}(y) \in T(y)$ where $T(y)=\{\pi(y) \cdot z=0\}$.

The proposition is derived in a pedestrian way in appendix 1 , in which the mappings $\lambda_{\ell}, \gamma_{\ell}$ are carefully defined and assessed.

We may illustrate the above concepts as well as our first insights in very simple cases.

The first case has only one factor $K=1$, and 2 goods, $L=1,2$, the production of which has constant returns to scale. The case is both well known and easy to visualize, as in Figure 1.

Figure 1 depicts the transformation possibilities of sector 1, (resp.2) when it can use one unit of the scarce factor, in the quadrant underneath, (resp. to the left) of the positive quadrant. When the unit of scarce factor can be shared in proportions $t, 1-t$, the corresponding production plan obtains as the convex combination of two production plans in the left and right quadrant. The straight line $\mathrm{AB}$ visualizes the upper limit of attainable production plans.

In the above formal terms, Figure 1 visualizes, the section of the global production set, a subset of $R^{L+K}=R^{3}$, with the hyperplane $v=1$, i.e attainable global 


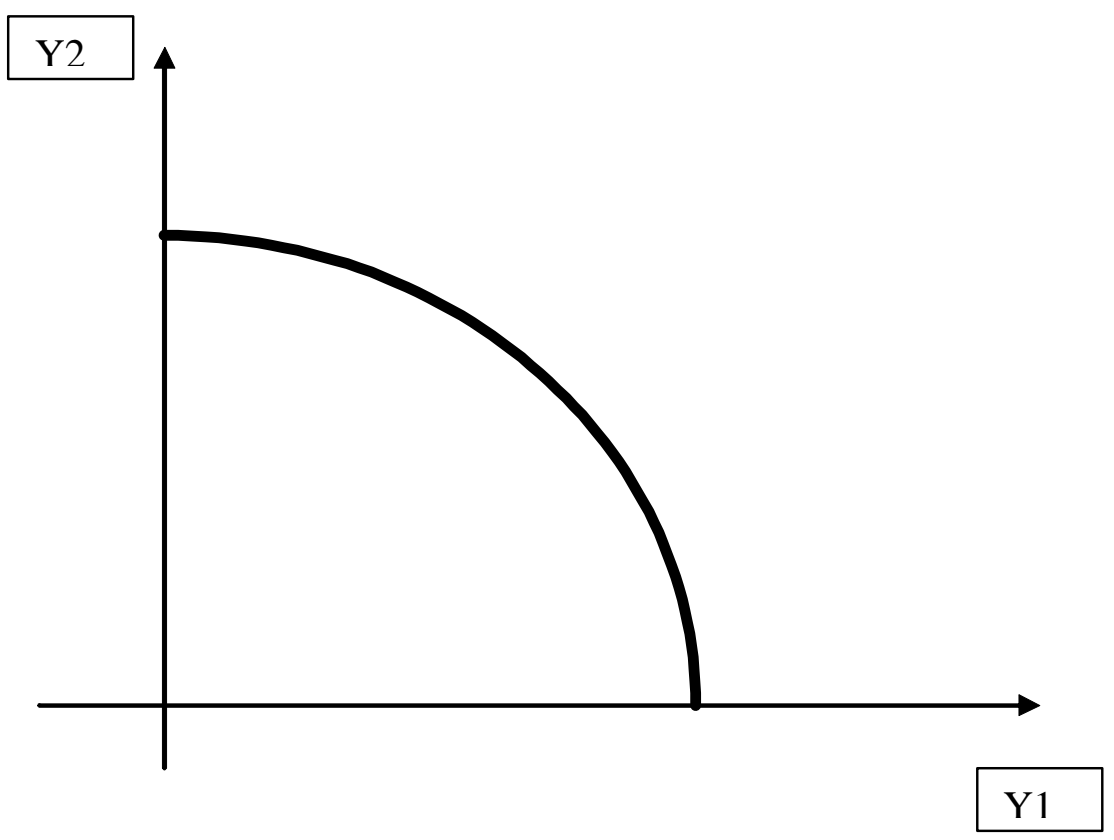

production plans when the available quantity of factor is 1 (what we called $Y(.1)$ ). The production set itself is the cone generated in $R^{3}$ from this section. The efficient frontier of $Y, Y_{E}$, is the cone in $R^{3}$ generated by the upper bold line (the upper bound of the section). The efficient frontier of $Y_{N S}, Y_{N S E}$ is the cone in $R^{3}$ generated from the segment $\mathrm{AB}$ of the straight line : it is a portion of hyperplane. This is a remarkable and well known property, on which we come back later.

Note that this primal view of the efficient set has a dual counterpart : there is a single supporting hyperplane $T(y)$ to the efficient part of the non-specialized global production set : also, the associated production prices, $\pi(y)$ are proportional to the "labour values" of goods

Such a geometry is not general: a two goods and two factors model of HeckscherOhlin type, the global production set is obviously still a cone. Its section by the plane $\left(v_{1}=1, v_{2}=1\right)$ would be again be convex, but it would be strictly convex, so that the boundary of its section in the positive quadrant would not be a straight line, as in Figure 1, but a single point, as in Figure 2. ${ }^{12}$

\footnotetext{
${ }^{12}$ This is well known in the standard H-O model; in our version of the model, it may be proved directly but also results for example from the forthcoming theorems.
} 


\section{Global economic efficiency :}

\subsection{The primal viewpoint and the geometry of $Y_{E}$.}

The geometry of $\mathbf{Y}_{N S E}$. Let us now focus attention on the non specialized efficient frontier $Y_{N S E}$. Remind here the examples of Section 2

We already know from the decentralization lemma that $Y_{N S E}$ is smooth, in the sense that at every point $y$ of $Y_{N S E}$ there is a unique normalized supporting remuneration plan $\pi(y)$, and hence a well-defined and unique tangent hyperplane $T(y)$. The next result tells us that $Y_{N S E}$ is made up of $L$-dimensional cones, smoothly glued together.

Take an efficient NS production plan $y$, and consider the (unique) normalized $y$-allocation $\left(\gamma_{\ell}(y), \lambda_{\ell}(y)\right), 1 \leq \ell \leq L$. Set:

$$
C(y)=\left\{\sum_{\ell} \mu_{\ell} \gamma_{\ell}(y) \mid \mu_{\ell}>0,1 \leq \ell \leq L,\right\}
$$

$C(y)$ is an $L$-dimensional polyhedral cone in $R^{K+L}$, with vertex at 0 , and edges carried by the $\gamma_{\ell}(y)$. In the case $K=1, L=2$ we investigated before, $C(y)$ is the two-dimensional cone in $\left(x_{1}, x_{2}, v\right)$-space, the section of which with $v=1$ is the line segment we depicted in Figure 1.

We know that $y \in C(y)$ (just take $\mu_{\ell}=\lambda_{\ell}(y)$ ). Recall, from the previous section, that $T(y)=\left\{y^{\prime} \mid \pi(y) y^{\prime}=0\right\}$ is the tangent hyperplane to $Y$ at $y$.

Proposition 3. $C(y)=T(y) \cap Y_{N S E}$.

The proof is in appendix.

The proposition provides the first "primal" insight into the geometry of the set $Y_{N S E}$ : it is a disjoint union of L-dimensional polyhedrals, each of one being the intersection of some tangent hyperplane with the set $Y_{N S E}$ itself.

This property will not be commented here at his stage. Note only that in both cases illustrated in Figure 1 and 2, $Y_{N S E}$ consists of a single 2-dimensional polyhedral. We have already commented the property on Figure 1, although its illustration through Figure 2 requires some additional mental gymnastics.

Non-substitution theorems. Remind that, for $v \in-R_{++}^{K}, Y(., v)=\{x \mid(x, v) \in Y\}$ and, with similar notations, we have defined $Y_{N S}(., v), Y_{N S E}(., v)$, (all are convex sets with non-empty interior, but not cones) and $Y_{N S E}^{+}(., v)$.

A facet of $Y_{N S E}(., v)$ is the intersection of $Y_{N S E}(., v)$ with a tangent hyperplane; it is necessarily a closed convex set containing the points of contact. We define its 
dimension to be the dimension of the smallest affine subspace which contains it, and we recall that its relative interior is its interior relative to its affine span.

Our main result in this subsection, which generalizes the classical non-substitution theorems, is :

Theorem 4. Every $x \in Y_{N S E}(., v)$ belongs to a single facet. This facet has dimension $(L-K)$, and $x$ belongs to its relative interior.

The proof is in appendix .

There are two limiting situations, $K=1$ (one factor) and $K=L-1$ (many factors). In the first case, we get the classical non-substitution theorem of Arrow and Samuelson:

Corollary 5. When there is only one factor of production, available in total quantity $-v \in R_{++}^{K}$, the set $Y(., v) \cap R_{+}^{L}$ of all goods bundles which can be produced is bounded by an affine hyperplane.

Proof. The set $Y(., v) \cap R_{+}^{L}$ is bounded by $Y_{N S E}(., v) \cap R_{+}^{L}$. By Theorem 4, every $y \in Y_{N S E}(., v)$ belongs to a single $(L-1)$-dimensional facet, and is in its relative interior. The only possibility is that this facet is common to all $y \in Y_{N S E}(., v) \cap R_{+}^{L}$ , and the result follows.

To say what happens in the second case, we must recall that a hypersurface in $R^{L}$ is ruled if it is a union of straight lines, and that it is developable if it is ruled and, along each of these straight lines, all points have the same tangent hyperplane. The next corollary does not seem to have appeared in previous literature, although it would (probably) follow from Travis (1972?) in the case $L=3, K=2$, and from (2006) in the case $L=4, K=3$

Corollary 6. If there are $L-1$ factors of production, available in total quantity $-v \in R_{++}^{K}$, the set $Y(., v) \cap R_{+}^{L}$ of all goods bundles which can be produced is bounded by a developable hypersurface.

Corollary 6 is visualized on Figure 3, for $L=3, K=2$. The figure provides a good support for intuition of the general situation.

It is suggestive in showing why the dimension of the faces, here one, is compatible with the fact that supporting prices vary smoothly on the efficiency surface. This is a key point in the general understanding of the problem.

Another interesting case is $L=2 K$ : there are twice as many goods as factors. Corollary 6 provides another viewpoint on the general situation, in what will appear later (Section ..) a borderline case 


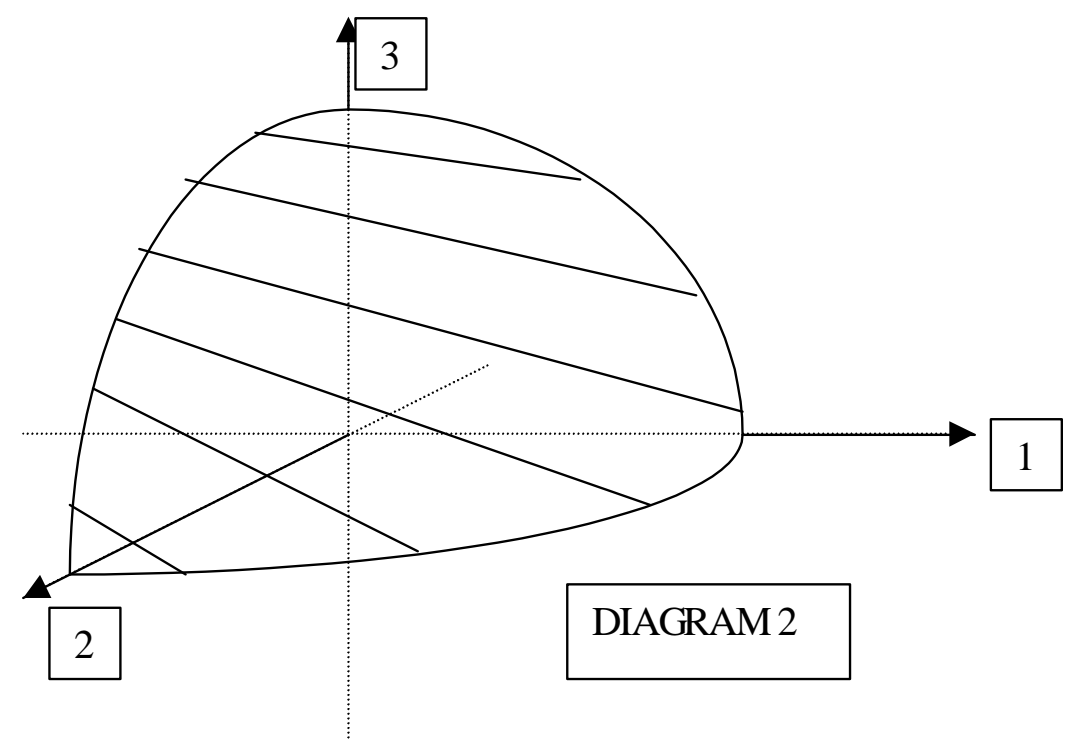

Corollary 7. If $K=L / 2$, every $x \in Y_{N S E}(v) \cap R_{+}^{L}$ belongs to the relative interior of some $K$-dimensional facet of $Y(v) \cap R_{+}^{L}$.

Corollary 7 is another consequence of Theorem 4 : the result was stressed first by Mc Kenzie (1955)

Corollary 8. (Mc Kenzie) : If $\left(p, w_{1}\right),\left(p, w_{2}\right)$ are two remuneration plans in $Y_{N S E}(v)$ associated with $x, x^{\prime}$, then $w_{1}=w_{2}$.

Proof. Proof : McKenzie's proof (in a model with a finite number of activities and hence slightly different from this one) would however works here. The result is here a simple consequence of the above theorem : suppose that there exist such $x, x^{\prime}$, in two different facets. The statement implies necessarily : $p x+w_{1} v>p x^{\prime}+w_{2} v$, so that $p x>p x^{\prime}$. But symmetrically one would have $p x^{\prime}>p x$, a contradiction.

This is the first occurrence in the paper of what we shall call later (Section ) a factor price equalisation result, : Two different facets in $Y_{N S E}(v)$ have different normal price vectors $p$.

The geometry of $Y(., x)$. Remind that : $Y(x,)=.\left\{v^{\prime} \mid\left(x^{\prime}, v^{\prime}\right) \in Y, x^{\prime} \geq x\right\}$ the set of factor endowments that allow to produce $x$,

We have : 
Proposition 9. The set $Y(x,)=.\left\{v^{\prime} \mid\left(x^{\prime}, v^{\prime}\right) \in Y, x^{\prime} \geq x\right\}$, is strictly convex, $\forall x \in$ $\operatorname{Pr} \operatorname{oj}_{x} Y$

Proof. The proof will be given later, but the statement is more conveniently presented unde the present headings

It follows :

Corollary 10. The sets $Y\left(e^{l},.\right) \forall l=1 \ldots L$, are strictly convex.

3.2. Efficiency : the dual viewpoint. Dual characterisation of efficiency has already being presented. The dual viewpoint leads us to describe the efficiency frontier indirectly, through the geometry of the set of supporting prices (mathematically speaking, we describe the polar cone of the production set rather than the cone itself). We pursue the implications of the initial analysis by emphasizing the problem of cost minimization.

Cost Minimisation, production prices, and the "factor values" matrix.. Let a remuneration plan $q=(p, w) \in R_{++}^{L+K}$ be given. Say we wish to produce one unit of good $\ell$. To do it at minimal cost requires solving the optimization problem:

$$
\operatorname{Min}\left\{p x^{-\ell}+w v \mid x^{-\ell} \in R_{+}^{L-1}, \quad v \in R_{+}^{K}, \quad f_{\ell}\left(x^{-\ell}, v\right) \geq 1\right\} .
$$

It follows from our assumptions that this problem has a unique solution ${ }^{13}$ for every $q$. In other words, there is a unique cost-minimizing bundle of goods and factors for the production of one unit of good $\ell$. In line with the notation of formulas $(22),(23)$ and (24) we denote this solution by:

$$
\left(a_{\ell}(p, w), b_{\ell}(p, w)\right) \in R_{++}^{L} \times R_{++}^{K}
$$

with $a_{\ell}^{\ell}=0$. We shall refer to the $\left(a_{\ell}^{j}, b_{\ell}^{k}\right)$ as the Leontieff coefficients associated with the remuneration plan $q=(p, w)$. The unit production cost of $\operatorname{good} \ell$ is then

$$
c_{\ell}(p, w)=p a_{\ell}(p, w)+w b_{\ell}(p, w)=\sum_{j \neq \ell} p_{j} a_{\ell}^{j}(p, w)+\sum_{k} w_{k} b_{\ell}^{k}(p, w)
$$

which is the optimal value achieved in problem (5). It follows from Conditions 1 and 2 that the function $c_{\ell}$ is positively homogeneous of degree one and concave. Using the nondegeneracy in Condition 3 and the inverse function theorem, we see that it is $C^{1}$ as well.

\footnotetext{
${ }^{13}$ Indeed, existence follows from the fact that the set where $f_{\ell}\left(x_{-\ell}, v\right) \geq 1$ is convex and contained in the strictly positive orthant, by conditions 1 and 2 , while the coefficients of $(p, w)$ are strictly positive. Uniqueness follows from condition 3 and the strict quasi-concavity of $f_{\ell}$
} 
In a remuneration plan, production prices must be equal to costs, (average and marginal costs are equal in our constant returns to scale setting) so that the following relation holds :

$$
p_{\ell}=c_{\ell}(p, w)=p^{\prime} a_{\ell}(p, w)+w^{\prime} b_{\ell}(p, w), \forall \ell
$$

In order to describe this system of equations in a more compact way, let us denote by $A(p, w)$ the $L \times L$ matrix with $a_{\ell}(p, w)$ as $l$-th column (so that all diagonal coefficients are zero) and by $B(p, w)$ the $L \times K$ matrix with $b_{\ell}(p, w)$ as $l$-th column. Considering $p$ and $w$ as line vectors, equations (8) then become:

$$
p(I-A(p, w))=w B(p, w)
$$

If the above formula holds true for some $p \in R_{++}^{L}$ and $w \in R_{++}^{K}$, then $A^{\prime}(p, w)$ is necessarily productive, and the same is true of its transpose ${ }^{14} A(p, w)$ so that the matrix $I-A(p, w)$ is invertible with positive entries. Hence, one will write (9) as :

$$
\begin{gathered}
p=w B(p, w)(I-A(p, w))^{-1} \\
=w\left[B(p, w)+B(p, w) \sum_{n \geq 1} A^{n}(p, w)\right] \\
p=w B(p, w)(I-A(p, w))^{-1}=w\left[B(p, w)+B(p, w) \sum_{n \geq 1} A^{n}(p, w)\right]
\end{gathered}
$$

Note then, that if the bundle $x$ has to be produced with the techniques $B, A$, the vectors of factors directly required for production is $B x$, the vectors of goods directly required is $A x$, and the vector of factors indirectly required for producing these goods is $B A x$, and the quantity of goods indirectly required is $A^{2} x$, the production of which also requires $B A^{2} x$ of factors, and so forth.

In a one-dimensional setting $(K=1)$, where the only factor is called labour, this formula expresses the standard finding that the labour value of a commodity is the sum of direct labour plus (total) labour indirectly incorpated in the production of the commodity.

Let us denote $F(p, w)=B(p, w)(I-A(p, w))^{-1}=\left[B(p, w)+B(p, w) \sum_{n>1} A^{n}(p, w)\right]$.

Here, from the decomposition just stressed the term $F_{k l}$ at the kth line and $l$ th column of the $K-L$ matrix $F(p, w)$ is similarly interpreted as the total quantity of factor $k$ used directly and indirectly in the production of good $l$, (with the cost minimizing techniques when the inputs prices are $(p, w))$. The $k t h$ line of the matrix is a one-dimensional line vector describing the factor $k$ value of the $l$ goods : it is the "labour values" vector, when the factor is called labour. Similarly, the $l$ th column is

\footnotetext{
${ }^{14}$,See for example Horn-Johnson (1995), Green-Whinston-Mas-Colell ()
} 
the vector of factors directly or indirectly required for the production of one unit of $\operatorname{good} l$,

We propose to call $F$ the "factor values" matrix.

Cming back on the fact that if $(p, w)$ is a remuneration plan associated with some efficient production plan in $Y_{N S E}$, then $p=w F(p, w)$. we reassess it in words :

The price of any good equals the sum over factors of the price of the factor multiplied by the factor value of the good.

Relating the primal and the dual viewpoint.. The proof of next result

requires a careful comparison of the dual and primal notation which is made in the appendix. We rewrite the findings of proposition 9 and 10 of the appendix, in a way that is immediately relevant here.

Proposition 11. 1-If $y=(x, v) \in Y_{N S E}$, and $(p, w)=\alpha \pi(y)$ is an associated remuneration plan, then $: p=w F(p, w)$, and $\exists \lambda \geq 0$ s.t $x=(I-A(p, w) \lambda, v=B(p, w) \lambda$

2- If . $(p, w) \in R_{++}^{L+K}$, and $p=w F(p, w)$, then any $y=(x, v), x \in R^{L}, v \in-R_{++}^{K}$, such that there exists $\exists \lambda \geq 0$ with $x=\left(I-A(p, w) \lambda, v=B(p, w) \lambda\right.$, belongs to $Y_{N S E}$, and $(p, w)=\alpha \pi(y)$.

Corollary 12. In both parts 1 or 2 of Proposition 1, one has $F(p, w) x+v=0$.

In addition to the factor values interpretation of efficiency prices stressed again in the proposition, the corollary says that for any efficient production plan, the factor values of a good vector in equals the quantity of factors available for production. The latter property obtains from the decomposition of $y$ stressing activity levels $\lambda$, s.t $\lambda[I-A().] \lambda=x$ and $B(.) \lambda=-v$, as noted in parts 1 and 2 of the proposition, and derived and expressed carefully in the Appendix.

\section{PRICES OF FACTORS AND PRICES OF GOODS}

At this stage, it is clear that pursuing the dual viewpoint require that we improve our inderstanding of the structure of the set of all possible remuneration plans $q=(p, w)$.

4.1. - A key insight, the Preparation theorem.. Let us come back to the above pricing equations, that characterize the couples $(p, w)$ that are remuneration plans:

$$
p=w B(p, w)(I-A(p, w))^{-1}=F(p, w) .
$$

It has been noticed early, that a standard counting, number of equations number of unknowns, suggests that whenever the number of goods equal the number of factors, there may be a one-to-one correspondance between the factor price and the goods prices. Also, with more goods than factors, the same unformal counting seems to 
reinforce the presumption. In fact, neither the first "finding" nor the second one are true. The examples of Section 5 illustrate this remark to which we come back later.

However, behind these unformal counting exercises, there is something which is general and true and which is indeed captured by the implicit function theorem : if $(p, w)$ is a solution of the above equations, and if we change $w$ by $d w$, then, there exists a unique $p+d p$ that satisfies the equations. In other, the set of $(p, w)$ that satisfies "locally" the equations is such that $p=\varphi(w)$; in more technical words, the set is the "local" graph of some function $\varphi$. We show here that this function can be extended to a global one.

Let us be more formal.

The set under scrutiny in this section is the cone ${ }^{15}$ :

$$
\begin{gathered}
\Sigma=\left\{q=(p, w) \mid q=\mu \pi(y), \mu>0, y \in Y_{N S E}\right\} \\
\Sigma=\left\{q=(p, w) \in R_{++}^{L+K} \mid p=w B(p, w)(I-A(p, w))^{-1}\right\}
\end{gathered}
$$

.We begin, as announced, by showing that $\Sigma$ is locally the graph of a map $\varphi$. This local property has been known in the literature of the seventies and eigthies although rarely stressed as such. ${ }^{16}$

Lemma 13. Let $q=(p, w) \in \Sigma$. There exists a neighbourhood $\mathcal{N}$ of $w$ in $R_{++}^{K}$, and a smooth map $\varphi: \mathcal{N} \rightarrow R^{L}$ such that $\varphi(w)=p$ and:

$$
\{(\varphi(t), t) \mid t \in \mathcal{N}\} \subset \Sigma
$$

Proof. Let $q=(p, w) \in \Sigma$. So equations (12) hold true in a neighborhood of $\Sigma$. By the envelope theorem, this set of equalities can be differentiated as :

$$
d p=B(q)(I-A(q))^{-1} d w
$$

The conclusion follows from the implicit function theorem applied at $q$.

\footnotetext{
${ }^{15}$ Recall that $\pi\left(Y_{N S E}\right) \subset E_{1}$, meaning that $p_{1}$ has been normalized to 1 . Equivalently :$$
\Sigma=\left\{q=(p, w) \mid q=\mu q^{\prime}, \mu>0, q^{\prime} \in \pi\left(Y_{N S E}\right)\right.
$$

The fact that this set is non-empty follows from $\mathrm{C} 4$.

${ }^{16}$ See however Woodland (1983), chapter 5 and specially p. 115,116 .

Naturally, in the case where goods are produced only from factors (as is the case in the GaleNikaido tradition evoked below) both the local and the global property have been known for long : $\varphi$ consists of the collection of standard cost functions (costs depending only on $w$ ) and from standard production theory,
} 
This defines $\varphi$ locally only. We are going to show that it can be defined globally. More precisely, we will show that the projection map from $\Sigma$ to $R_{++}^{K}$ is globally oneto-one. The proof here is more delicate, and is given in Appendix 2. We show first that above each $w$ there can be at most one $p$ (Lemma ?? $)^{17}$, and then that the local extension can be made global (Lemmas ?? and ??).

Theorem 14. (Preparation Theorem) $\Sigma$ is the graph of a map $\varphi: R_{++}^{K} \rightarrow R_{++}^{L}$ , smooth and homogeneous of degree one.

In economic terms, the theorem asserts that prices of factors globally determine the prices of goods. Although the statement is straigthforwardly established in models where goods are produced from factors, we believe it si novel in the present context..

It follows immediately that the relative prices of factors globally determine the relative prices of goods. Normalizing the prices of goods by setting $p_{1}=1$ (that is, taking the intersection with $E_{1}$ ) tells us that the prices of factors should satisfy $\varphi(w)_{1}=1$. In other words, $w$ should belong to the $(K-1)$-dimensional manifold $F_{1}$ defined by the nonlinear equation:

$$
\left[(I-A(\varphi(w), w))^{-1} B(\varphi(w), w) w^{\prime}\right]_{1}=1
$$

where the notation []$_{1}$ denotes the first component of the bracketed vector.

Next, we have :

Proposition 15. The jacobian derivative of $\varphi: R_{++}^{K} \rightarrow R_{++}^{L}$, a smooth and homogeneous of degree one map is

$$
\partial \varphi(w)=F(\varphi(w), w)
$$

Where $F$ is the $L-K$ matrix of transposed "factor values" matrix, evaluated in $(\varphi(w), w)$

Proof. It is an easy application of the "enveloppe theorem".

In words, the property has a very intuitive interpretation, when taking into account our interpretation of the "factor value" matrix : the change of the price of good $l$, induced by a (small) change of the price of factor $k$ (approximately) equals the factor $k$ value of good $l .^{18}$

\footnotetext{
${ }^{17}$ The argument is reminiscent of a standard argument in the analysis of the Malinvaud-Taylor algorithm, see Malinvaud (1967). We owe the idea as well as relevant references to Michael Jerison.

${ }^{18}$ In the case wher goods are produced only from factors, it is clear that $\partial \varphi(w)$ obtains from the collection of cost minimizing input bundles : the factor $k$ value of good $l$ is there the direct quantity of factor $k$ used for the production of one unit of good $l$
} 
Note that, specifiyng the above statement in a $2 x 2$ world, leads to the StoplerSamuelson theorem. It may make sense to view this formula as a generalized StoplerSamuelson theorem. ${ }^{19}$.

4.2. A synthetical dual description.. Up to now, we have focused attention on sections with fixed $v$ asking ourselves what are the remuneration plans, which we may index by $w$. A natural question is to know what is the set of $w$ associated with a given $v$ or, equivalently, the set of $v$ associated with a given $w$ ? Next proposition sheds light on the second question.

Proposition 16. Fix $w$. The set of $v$ such that $(\varphi(w), w)$ is a remuneration plan for some $y$ in $Y_{N S}$ is a closed convex cone denoted $K(w)$ generated by the vectors $F_{l}(\varphi(w), w), l=1 . . L$. The mapping $w \rightarrow(K(w))$ is continous.

The knowledge of the mapping $K(w)$ together with the mapping $\varphi(w)$ leads to a simple synthetical description of the efficiency frontier under scrutiny here. Let us provide it first from the sections $Y_{N S E}(., v)$

Theorem 17. Description of $Y_{N S E}^{+}(., v)$.

The knowledge of $\varphi(w), K(w)$ determines $Y_{N S E}^{+}(., v)$ as follows.

$Y_{N S E}^{+}(., v)=\cap_{w \in K^{-1}(v)}\left\{x^{\prime} \in R_{++}^{L} \mid F(\varphi(w), w) x^{\prime}+v=0\right\}$.

Theorem 17 puts emphasis on $Y_{N S}^{+}(., v)$, but its proof has implications on the description of $Y_{N S E}$. that are displayed as Corollary 18. Both will be commented later.

Corollary 18. Description of $Y_{N S E}$.

$$
\begin{aligned}
& \varnothing \neq \cap_{w \in K^{-1}(v)}\left\{\left(x \in R_{++}^{L}, v\right) \mid F(\varphi(w), w) x+v=0\right\} \subset Y_{N S E} \\
& Y_{N S E} \subset \cap_{w \in K^{-1}(v)}\{(x, v) \mid F(\varphi(w), w) x+v=0\}
\end{aligned}
$$

The proof of the above statements relies on a series of Lemmas

Lemma 19. The set of $v$ for which there is a facet contained in a set $\left\{x^{\prime} \mid F(p, w) x^{\prime}+\right.$ $v=0\}$ for a given $(p, w)$ is a convex cone $K(p, w)$.

Proof. Take $(p, w)$ and $y^{1}=\left(z^{1} v^{1}\right), y^{2}=\left(z^{2}, v^{2}\right)$, such that $(p, w)=\alpha\left(y^{1}\right)=$ $\alpha\left(y^{2}\right)$, then $\{F(p, w) z+v=0\}$, for $\left(z^{1} v^{1}\right),\left(z^{2}, v^{2}\right)$ implies that it holds for $t z^{1}+(1-$ t) $z^{2}, t v^{1}+(1-t) v^{2}$.

\footnotetext{
${ }^{19}$ This formula also can allow to stress "friends" and "enemies" along the lines of Jones-Scheinkman (1977).
} 
Lemma 20. Consider $y=(x, v) \in Y_{N S E}$, and $(p, w)$ is an associated remuneration plan.Then, the $L$-dimensional cone $C(y)$ in $Y_{N S E}$ to which, according to Theorem 4, y belongs is such that : $\quad\left\{\left(x^{\prime} \in R_{++}^{L}, v^{\prime}\right) \mid F(p, w) x^{\prime}+v^{\prime}=0\right\} \subset C(y)$

$$
C(y) \subset\left\{\left(x^{\prime}, v^{\prime}\right) \mid F(p, w) x^{\prime}+v^{\prime}=0\right\}
$$

and the facet in $Y_{N S E}(., v)$ to which it belongs is such that :

$$
\text { Facet }(x) \cap R_{++}^{L}=\left\{x^{\prime} \in R_{++}^{L} \mid F(p, w) x^{\prime}+v=0\right\}
$$

Proof. From the above proposition 11 and its corollary, we know that $F(p, w) \cdot x+$ $v=0$.From the same proposition, every $y^{\prime}=\left(x^{\prime}, v^{\prime}\right)$ in $Y_{N S E}$ with $(p, w)$ as remuneration plan is such that: $F(p, w) \cdot x^{\prime}+v^{\prime}=0$. Reminding that $C(y)=T(y) \cap Y_{N S E}$ and that $y^{\prime}=\left(x^{\prime}, v^{\prime}\right) \in T(y)=T\left(y^{\prime}\right)$, (normal to $(p, w)$ ), we get the second conclusion. For getting the first conclusion, we note that if $F(p, w) x+v=0$, for some $v$ and some $x$, then there is an $x^{\prime} \in R_{++}^{L}$ with $F(p, w) x^{\prime}+v=0$, The third conclusion follows easily from the definition of $Y_{N S E}(., v)$

At this stage, the proof proceeds as follows :

Proof. We then refer to the preparation theorem and the above lemma. If $F(\varphi(w), w) x+$ $v=0$, for some $v$ and some $x$, then there is an $x^{\prime} \in R_{++}^{L}$ with $F(\varphi(w), w) x^{\prime}+v=0$, so that $v$ does belong to the convex cone generated by the columns of $F(\varphi(w), w)$. Conversely, if $v$ belongs to this cone, the above lemma yields that it belongs to $K(w)$. Hence, the conclusion. The continuity properties of the mapping $K$ follow easily. Proposition 16 follows.

Theorem 17 then obtains from Proposition 16 and the lemma.

Theorem 17 provides the "equations" determining the cones of $Y_{N S}$ and the facets of $Y_{N S}(, . v)$ exhibited Proposition 3 and Theorem 3. In words, paraphrasing Theorem 4, the equations determining a facet in $Y_{N S}(, . v)$ read : the "factors values" of any production plan of goods in the facet equals the quantity of available factors.

The statement is clearly illustrated from previous figure 3. Each of the lines that generate $Y_{N S E}^{+}(., v)$ in the figure is the intersection of two hyperplanes, the first one expressing that, say, the total "labor" values of the three goods equals available labour, the second one expressing, say, that the "land value" of the three goods bundle equals the available land.

Next Corollary confirms the assertion of Proposition 9.

Corollary 21. $Y(x,$.$) is strictly convex.$

Proof. The set does not contain any "line".

Then, we have : 
Theorem 22. Description of $Y(x,$.

$Y(x,)=.\left\{\cup_{w \in R_{++}^{K}}(F(\varphi(w), w)) x\right\}+R_{++}^{K}$

and $-F(\varphi(w), w)) x$ is the unique solution to $\min \left\{-w v^{\prime} \mid v^{\prime} \in Y(x,)\right.$.

We first ask ourselves what is the cost minimizing bundle of factors, at factor price $w$,for producing $x$. The answer is simple : the unique solution is to take $-v=$ $F(p, w) x$. Let us prove it.

Proof. Let $(x, v)$ be a NS efficient production plan, and $(p, w)$ the supporting remuneration plan. Then, $v=-F(p, w) x$ is the unique solution of the problem:

$$
\min \left\{-w v^{\prime} \mid v^{\prime} \in Y(x, .)\right.
$$

and the optimal value is p.x. Applying Lemma ??, we get:

$$
p x+w x=\max \left\{p x^{\prime}+w v^{\prime} \mid\left(x^{\prime}, v^{\prime}\right) \in Y\right\}=0
$$

As a consequence, we have:

$$
w x=\max \left\{w v^{\prime} \mid\left(x, v^{\prime}\right) \in Y\right\}=-p x
$$

which is the desired result by (??).

Conclusion then follows from the Preparation theorem and Corollary 21.

We stress two useful corollaries

Corollary 23. Dual description of the sets $Y\left(e_{\ell},.\right), 1 \leq \ell \leq L$,

1- $B\left(Y\left(e_{\ell},.\right)\right)=\left\{\cup_{w \in R_{++}^{K}}\left(F^{l}(\varphi(w), w)\right\}+R_{++}^{K}\right.$ is a strictly convex set.

$2-\varphi_{l}: w \in R_{++}^{K} \rightarrow w F^{l}(\varphi(w), w)$ is the surrogate concave homogenous of degree zero cost function,

3- $\left(\partial^{2} \varphi_{l} / \partial w^{2}\right)$ is a symmetric negative semi-definite $K x K$ matrix of rank $K-1$.

4- $Y_{N S}^{+}$can be recovered from $Y\left(e_{\ell},.\right), \forall l$.

Proof. Theorem 22 together with the Preparation Theorem imply 1 and the fact that $\left(\partial \varphi_{l} / \partial w\right)$ coincides with the second derivative of the function $w \rightarrow w \cdot F(\varphi(w), w) e_{\ell}$ (which is homogenous of degree one). So it must be symmetric and degenerate. As $\varphi_{l}$ minimizes the cost, it is a concave function with respect to $w$, so that $\left(\left(\partial^{2} \varphi_{l} / \partial w^{2}\right)\right.$ must be negative semi-definite. 4 follows from ...

Finally we stress static compartive results, by examining the local co-movements of a NS production plan and the supporting remuneration plan. The following proposition summarizes the result in a way that attempts to compromise rigor and readibility. 
Proposition 24. ?? Let $\bar{y}=(x, v)$ be a NS efficient production plan, and $(p, w)$ some supporting remuneration plan, so that $p=\varphi(w)$ Then $(x+d x, v+d v)$ still belongs to $Y_{N S E}$, and $(p+d p, w+d w)$ still is an associated remuneration plan if and only if :

$1-p d x+w d v=0$,

$2-d p=(d w F[\varphi(w), w])$,

3- $d w M=d v-[F(\varphi(w), w)] d x$, where

$$
M:=\left.\frac{\partial}{\partial w}[F(\varphi(w), w) x]\right|_{w=\bar{w}} \mid
$$

is a symmetric negative semi-definite $K x K$ matrix of rank $K-1$.

Proof. 1 and 2 follow imediately from our previous findings ...

$>$ From the above theorem $M$ as a combination with strictly positive coefficents of $\left(\partial \varphi_{l} / \partial w\right)$ must be symmetric and degenerate. Note also that, by Proposition ??, $-F(\varphi(w), w) x$ is a cost-minimizing vector with respect to $w$, so that $M$ must be negative semi-definite.

Corollary 25. If $d w=0$, that is, if the prices of factors do not change, we get $d v=F(\varphi(w), w) d x$.

Ribzcinsky's theorem appears as a particular case of the above statement in a $2 x 2$ world with no-intensity reversal and where goods are produced from factors only.

\section{Factor Price Equalisation.}

The factor price equalisation preoccupation seems to have here a natural formulation, without predjudging at this stage how it interferes with the trade questions : in a given production economy, is it the case both that $\left(p, w_{1}\right)$ and $\left(p, w_{2}\right)$ can be remuneration plans so that the same price for goods is compatible with different prices of factors ? In other words, could it exist two distinct, here unconnected, economies with the same available technologies, two distinct total vector of factor endowments, such that respectively $\left(p, w_{1}\right)$ and $\left(p, w_{2}\right), w_{1} \neq w_{2}$,appear in equilibrium?

5.1. Classical findings : number of goods equal number of factors. This question has been looked at in the literature.

First, the question may be raised in different ways and has indeed been. For example Mc Kenzie asks a more demanding property : can it be the case that $\left(p, w_{1}\right)$ and $\left(p, w_{2}\right)$ can be remuneration plans, in the same section $v=$ ste of the production set. As we have seen, the answer has been known to be no, a theorem that is more easily recovered from the present analysis . In other words, one cannot find two similar economies, both in terms of technologies and inital endowments, in which the factor price equalisation fails. 


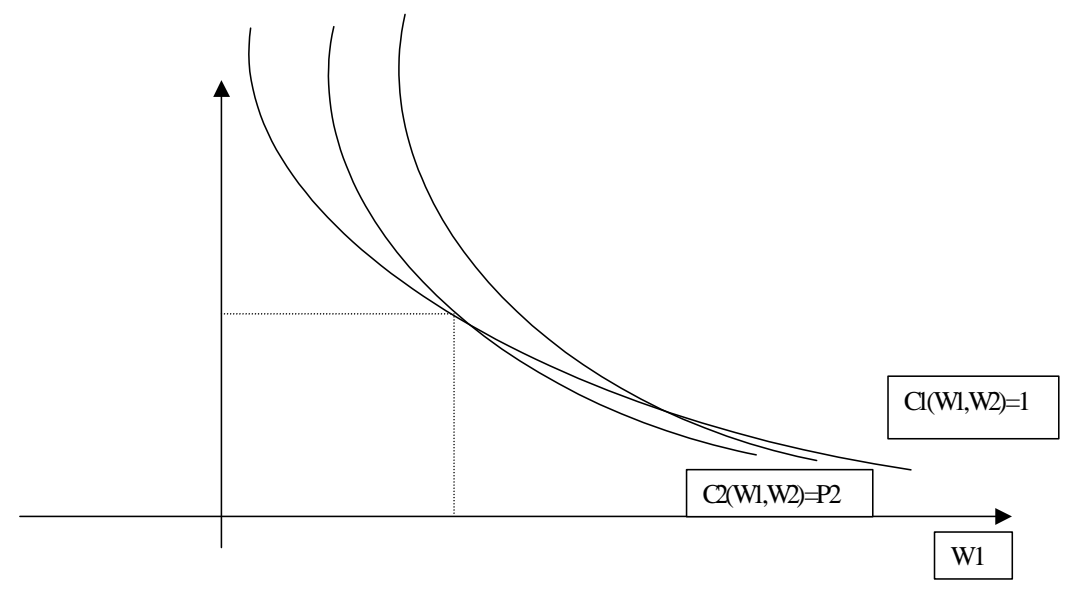

Let us come back to the initial and more difficult question : can $\left(p, w_{1}\right)$ and $\left(p, w_{2}\right)$ can be remuneration plans, (for different $v$ )

It has been known for some time that the answer to the above question is no in the two goods two factors models, whenever the so-called factor intensity of goods is not reversed in the production process : this is the no-intensity reversal condition. Under the no-intensity reversal condition, the (remuneration) price of goods uniquely

determine the price of factors : this is the factor price equalisation property. Indeed, in this case, in the plane $\left(w_{1}, w_{2}\right)$ of the prices of factors, which here is drawn under the assumption that there is no intensity reversal and that good 1 is more intensive in factor 1.

Such a univalence theorem has been extended to the case where the the number of factors equals the number of goods and goods are produced only from factors, in a literature starting from Gale-Nikaido (1965) and which was active in the seventies (see f.e Nikaido (1972)). The literature has emphasized a generalized no-intensity reversal condition, a weaker form of which appears in Mas-Colell (1979b).

We show that Mas-Collell's findings hold in the extended setting we consider here.

Assume that $K=L$ and consider the function $\varphi: R_{++}^{K} \rightarrow R_{++}^{L}$, whose existence has just been shown.

As in Mas Colell (1979b), let us consider the share matrix $S(\varphi)$ with entries:

$$
s_{\ell k}=\frac{w_{k}}{\varphi_{\ell}} \frac{\partial \varphi_{\ell}}{\partial w_{k}}
$$

As we have seen above, $S(\varphi)$ can be computed explicitly in terms of $A$ and $B$. 


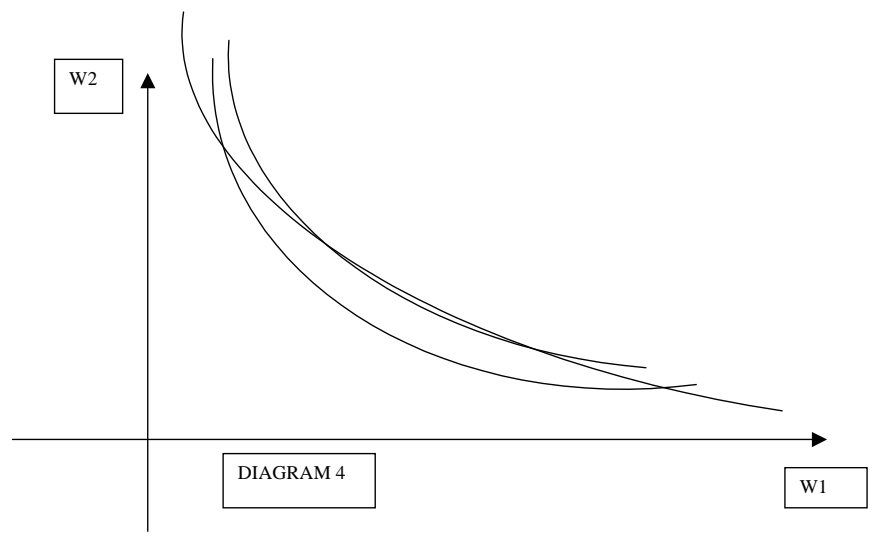

The extended non-intensity reversal assumption that we consider asserts that the determinant $(\operatorname{det} S)$ never vanishes : the reader will note that in the one-dimensional case this is equivalent to non intensity reversal as discussed above.

Corollary 26. Assume $K=L$, consider the function $\varphi$ the existence of which is established in the above preparation theorem, and assume that det $S \varphi(w)$ is bounded away from zero on $R_{++}^{K}$. Then $\varphi: R_{++}^{K} \rightarrow R_{++}^{L}$ is onto and one-to-one.

Proof. The assumption means that there is some $\varepsilon>0$ such that $|\operatorname{det} S \varphi(w)|$ $\geq \varepsilon$ for all $w \in R_{++}^{K}$. The proposition follows the fact that, once $\varphi$ is shown to exist, the proof, and indeed the statement of Mas Colell (1979b) applies here ${ }^{20}$

5.2. Factor price equalisation in general.. However, the univalence property does not hold true when the no-intensity reversal condition does not hold, even if $K=L$ : for example the next figure, which exhibits iso-cost curves in the space of the factor prices, when $K=L=2$,shows an example of non intensity reversal, which has nothing pathological. Note that here, as well as in the previous picture, the analysis exploits the fact - the generality of which is established by Theorem 11that the set of normalized (the normalization obtains by putting the price of good one equal to one) remuneration plans $(p, w)$ is a one-dimensional object (because there are only two factors) that may be indexed by the ratio of factor prices (the length of the factor price vector being determined by the price normalisation).

\footnotetext{
${ }^{20}$ For the reader's convenience, let us mention that the proof consists in considering the map $\psi$ : $R^{K} \rightarrow R^{L}$ defined by $\psi(u)=\log \varphi\left(\exp u_{1}, \ldots, \exp u_{2}\right)$. By assumption, the determinant of the Jacobian matrix $D \psi$ is bounded away from zero. By Hadamard's global version of the implicit function theorem, $\psi$ is onto and one-to-on (see also Mas Colell (1979a))
} 


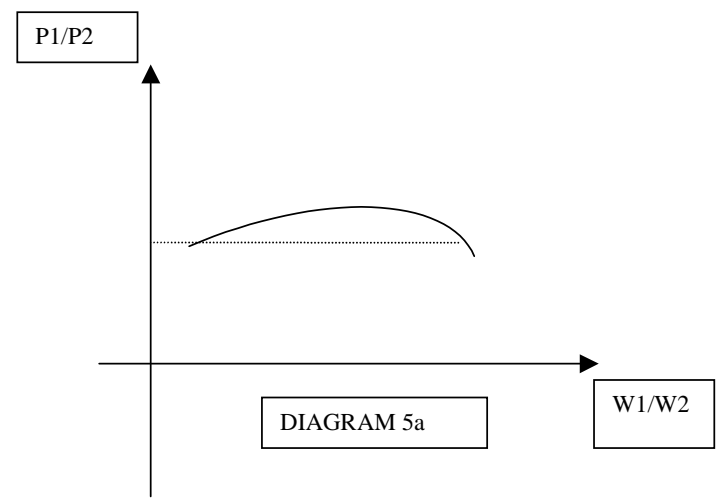

Here, the prices of goods do not any longer univoquely determine the prices of factors : every $p$ is associated with two different $w$. Therefore, plotting relative prices of goods in terms of relative prices of factors, we get a local maximum (or minimum), as in Figure 5.

Similarly, in the case where the number of goods were 3, with the same number of factors, $K=2, L=3$, mapping relative prices of goods in terms of relative prices of factors gives a map from $R$ into $R^{2}$ and Figure shows a picture with self-intersections. Each self-intersection corresponds to a point where the map is not one-to-one : there are some $p$ associated with two $w$. that is, the prices of goods in such a case do not determine the prices of factors. Although the picture rightly suggests that the non univalence situation is less frequent than in the previous example, it is non pathological (only strong assumptions as non intensity reversal can rule it out) and and (if it holds for some production economy, it holds for neighbour ones, for every reasonable definitions of neighbour). For example, in a three goods economy where there is intensity reversal occuring for every pair of goods, the reader is invited to visualize in the two dimensional factor prices where iso-costs functions are drawn, a situation where three iso-cost curves intersect in two different points and convince himself that the occurrence of the sitaution is not destroyed by a small change of the underlying production sector.

Note that a self-intersetion of the above curve in $w_{1}, w_{2} .$. satisfies $\varphi\left(w_{1}\right)=\varphi\left(w_{2}\right), \quad w_{1} \neq$ $w_{2}$

In a sense, we have two equations and two unknown, and the fact that this system of two equations has a solution should not be expected to be pathological. 

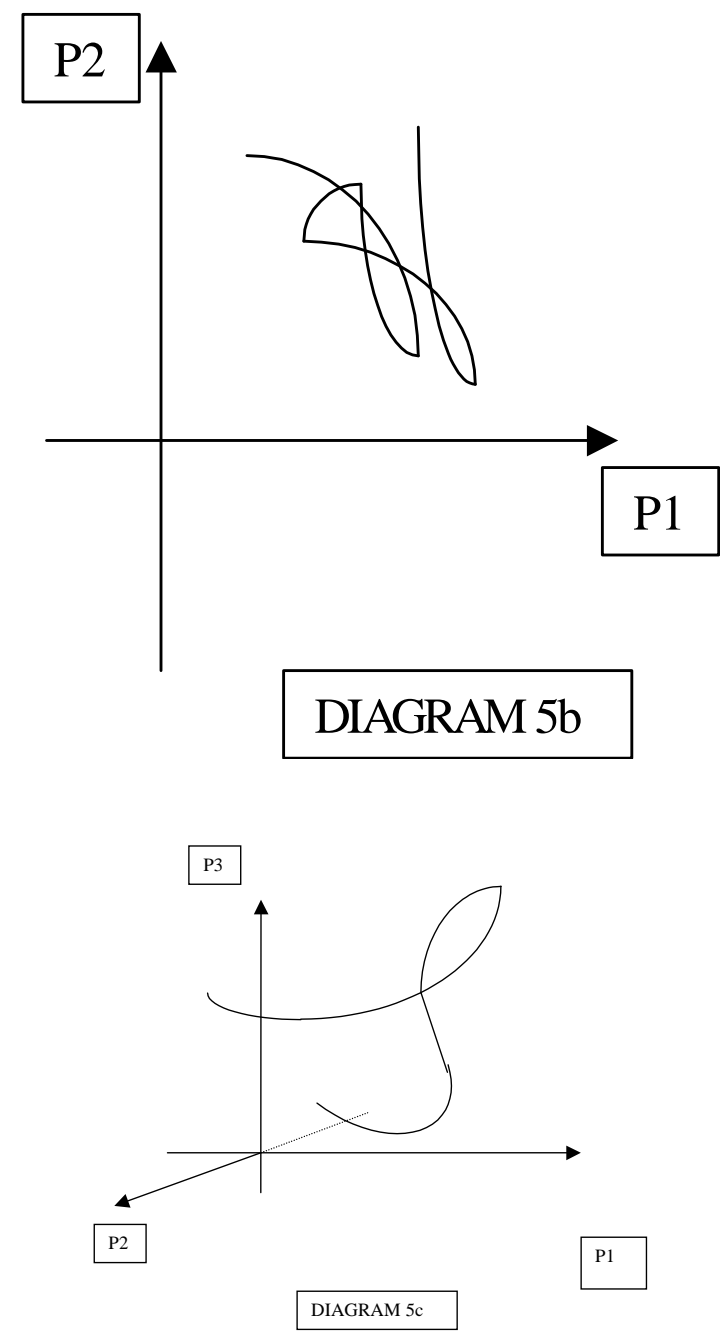

Coming back on the $K=2$ exemple, and reminding the one-dimensional propertyof the set of remuneration plans, we note that the likelihood of $p$ associated with two $w$, becomes weaker when $L=4$. With four goods, or more, the set under scrutiny would be a parametric curve in $R^{3}$, or in a higher-dimensional space, and it is extremely unlikely that such a curve would display self-intersections (see Figure $5 b)$.

We can also appeal to the above equations determining a self-intersection. The system $\varphi\left(w_{1}\right)=\varphi\left(w_{2}\right), \quad w_{1} \neq w_{2}$ has three equations and two unknowns.

Hence, the prices of goods should be expected to determine the prices of factors whenever $K \geq 4=2 L$. Indeed, one will show so that the factor price equalisation theorem holds. 
.The idea of the proof can be related with this counting of equations and unknowns. In the general case, there are $(L-1)$ equations, one for each (normalized) good price. On the other hand, there are $(K-1)$ unknowns for $w_{1}$ and $(K-1)$ for $w_{2}$, in total $2(K-1)$ unknowns. If there are more equations than unknowns, that is, if:

$$
\begin{aligned}
L-1 & >2(K-1) \\
L & >2 K-1
\end{aligned}
$$

Then a solution is unlikely.

5.3. A generic theorem. Using Thom's Transversality Theorem, is possible to frame the above discussion in a rigourous mathematical statement. Let us first note that a production economy, in our model, is entirely characterized by the family $f=\left(f_{1}, \ldots, f_{L}\right)$ of production functions. Pick some $r \geq 2$, and let us denote by $\mathcal{F}^{r}$ the set of all $f=\left(f_{1}, \ldots, f_{L}\right)$ which satisfies conditions C1 to C4, each $f_{\ell}$ being $r$ times continously differentiable. In Appendix 11 we prove the following:

Theorem 27. If $2 K \leq L$, then, there is generically in $\mathcal{F}^{r}$, factor prices equalisation holds in the economy generated by $f$

$$
\varphi\left(w^{1}\right)=\varphi\left(w^{2}\right) \Rightarrow w^{1}=w^{2} .
$$

In other words, for "almost all" $f \in \mathcal{F}^{r}$, factor prices equalisation holds in the economy where $f=\left(f_{1}, \ldots, f_{L}\right)$ are the production functions, whatever the total factor endowments. Since there is no equivalent of Lebesgue measure in infinite-dimensional spaces, such as $\mathcal{F}^{r}$ the expression "almost all" take on a special meaning, which is described in Appendix 11.

The proof of Theorem ?? is given in Appendix 11. It builds on the above intuition, that is, if there are more equations than unknown, then system (??) should have no solution, and if it happens to have one, it should be a pathological situation which could be corrected by an arbitrarily small perturbation of the function $\varphi$. But one can perturb $\varphi$ only through perturbing $f$, and the main difficulty in the proof is that the dependence of $\varphi$ on the $\left(f_{1}, \ldots, f_{L}\right)$ is not explicit. One then has to go through the implicit function theorem, and to prove that a certain number of determinants do not vanish, which gives the proof a rather technical character.

The idea of the proof can now be explained more precisely. Recall the definition of $E_{1}$ from (??) and $F_{1}$ from (13). Set:

$$
D=\left\{\left(w^{1}, w^{2}\right) \mid w^{1} \neq w^{2}\right\} \subset F_{1} \times F_{1}
$$




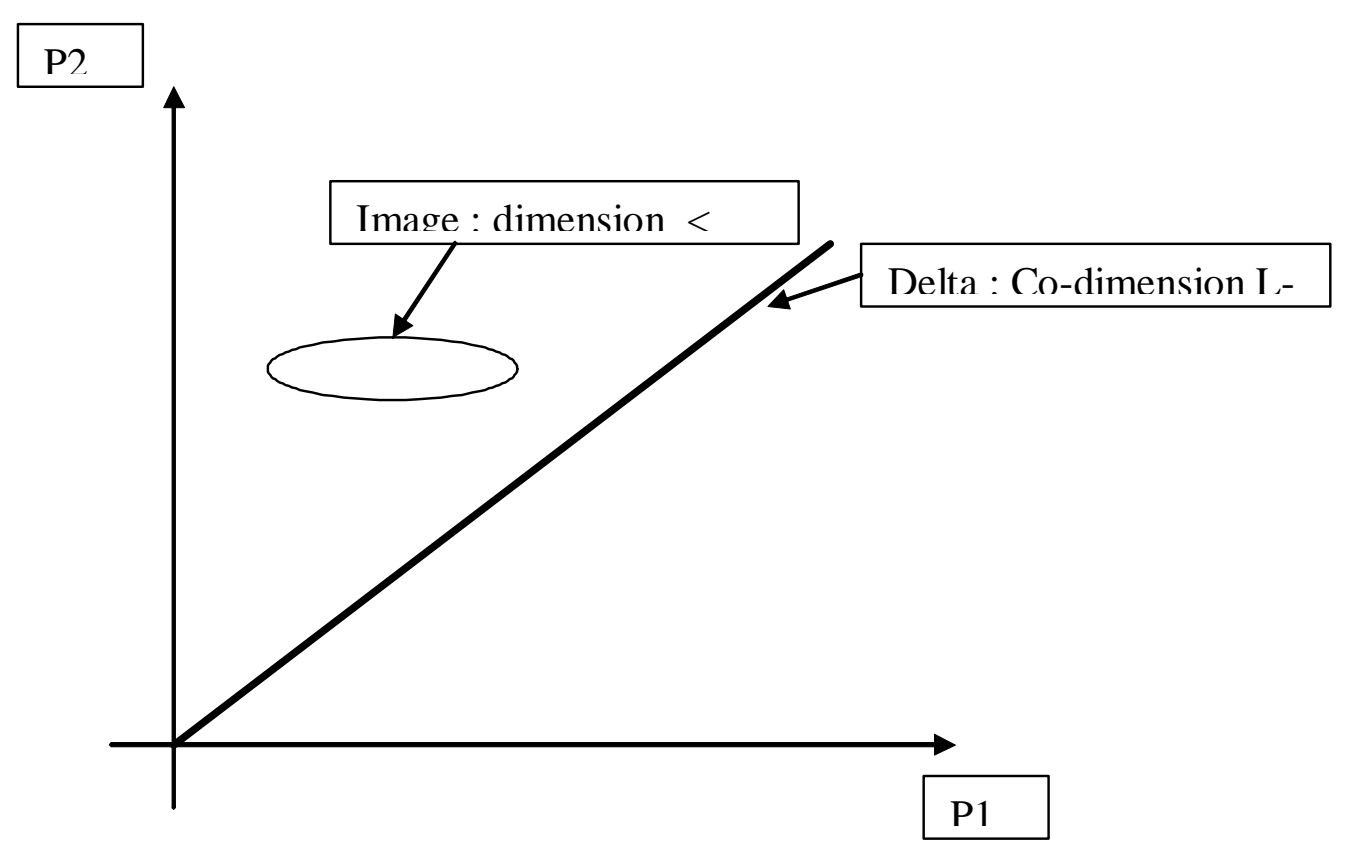

and define a map $\Phi: D \rightarrow E_{1} \times E_{1}$ by $\Phi\left(w^{1}, w^{2}\right)=\left(\varphi\left(w^{1}\right), \varphi\left(w^{2}\right)\right)$. Now consider the diagonal:

$$
\Delta=\left\{\left(p_{1}, p_{2}\right) \in E_{1} \times E_{1} \mid p_{1}=p_{2}\right\}
$$

Figure :

The codimension of $\Delta$ is $L-1$ (because $E_{1}$ has dimension $L-1$ ), and the dimension of the image of $\Phi$ is at most $2(K-1)$ (because $F_{1}$ has dimension $K-1$ ). If $2(K-1)<$ $L-1$, that is, $2 K \leq L$, the image of $\Phi$ will not intersect $\Delta$ in general ${ }^{21}$. This means that, when $2 K \leq L$, the equality $\varphi\left(w^{1}\right)=\varphi\left(w^{2}\right)$ cannot occur when $w^{1} \neq w^{2}$.

If $L<2 K$, there are two possible cases: $K<L<2 K$ and $L \leq K$. In the first one, we still have a factor price equalisation theorem, but a weaker one. It starts with the following geometrical result, which is also proved in Appendix 11.

Theorem 28. If $K<L<2 K$, generically in $\mathcal{F}^{r}$, the cone

$$
\mathcal{C}=\left\{\left(w^{1}, w^{2}\right) \in R_{++}^{2 K} \mid w^{1} \neq w^{2}, \varphi\left(w^{1}\right)=\varphi\left(w^{2}\right)\right\}
$$

\footnotetext{
${ }^{21}$ This is a nonlinear version of the geometrical fact that a randomly chosen linear subspace of dimension $p$ will not intersect another randomly chosen linear subspace of codimension $>p$.
} 
is either empty, or a submanifold of dimension $(2 K-L)$

Comments and corollary.

\section{A FEW WORDS ON APPLICATIONS.}

The direct applications of our results have been stressed (Corollary) or are obvious. However, there are many potential applications, some of them we have started to develop. We provide here a list of such applications. Our forthcoming paper (EkelandGuesnerie (2007)) attempts to describe more in detail the lines of research that may be pursued, while stressing some findings of work in progress.

Equilibrium theory with production. . Equilibrium theory of the walrasian type adresses many qualitative questions. We shall start here for the assesment of the Walras correspondence.

The properties of the Equilibrium correspondance. . The question is the following : Consider a set of economies - where endowments, preferences, etc...- may vary. The equilibrium correspondence associates with a given economy the set of equilibria of this economy : the knowledge of this correspondance brings a qualitative information : what is the number of equilibria, how does this number vary with the economy, in which cases is the number equal to one (the uniqueness question). At the highest abstract, as the one adopted by Kehoe (1985), the qualitative answers remain general, even by mobilizing the most powerful techniques.

In the setting of a many-consumers economy, where endowments $v_{h}$ in factors are privately owned and inelastically supplied, the comparative statics of the change from a situation 0 to a modified situation where preferences are fixed but individual initial endowments change by $d v_{h}$ is governed by an equation of the form : .

Comparative statics is the governed by the formula:

$M(0) d w^{\prime}=-(F(0))(\partial D / \partial p)_{0}^{C s t e} F(0) d w^{\prime}-\sum_{k} F(0) \sum_{h}\left(\partial D_{h} / \partial R\right)\left(v_{0, h}^{k}-F^{h} x_{0, h}\right) d w_{k}$

$\left.+\sum_{h} w_{0} d v_{h}\left(\partial D_{h} / \partial R\right)_{0}\right]+d v$.

where notation is the one adopted in the paper for $M, F, w, v$, possibly indexed by houselhold $v_{h}, x_{h}$ and where $(\partial D / \partial p)_{0}^{C s t e}$ denotes the matrix of compensated aggregate demand and $\left(\partial D_{h} / \partial R\right)$ the individual vectors of income effects.

The relevant matrix in a one consumer case is : $G(0)=\left\{A(0)+(F(0))(\partial D / \partial p)_{0}^{\text {Cste }}\left(F^{t}(0)\right)\right\}$ which is negative semi definite and invertible. The possible bifurcations are associated with the additional terms

$+\sum_{k} F(0) \sum_{h}\left(\partial D_{h} / \partial R\right)\left(v_{0, h}^{k}-F^{h} x_{0, h}\right) d w_{k}$

The difficulty is, like in an exchange economy linked to the individual income effects, that relate to the difference in the quantity of factor $k$ held by consumer $h$ and the quantity of factor $k$, directly or indirectly incorporated that he consumes. Heterogeneity then matters a lot... The understanding of this term is crucial for studying 
the bifurcations of the Walras correspondence, but also for other questions which are sketched here.

Others.. - Uniqueness : the just sketched analysis suggests new results that would idnetify connected components of economies without bifurcations and/or reflect limited heterogeneity of income effects. It also allows to appraise existing results obtained in the case where goods are only produced from factors and to extend them to the more complex setting.

- An alternative to the standard Walras tâtonnement would be a tâtonnement bearing on all (or a subset) of factor prices while supposing that the prices of the goods (in our teminology) are immediately equated to the price implied by the factor prices. One can find a number of arguments supporting this suggestion. One of them is that it was one of the original proposals of Walras himself (Walras (), p. ).

The proposition can be viewed formally as involving a tâtonnement process on an excess demand function that would be equal to $Z(w)=F(\varphi(w), w) D(\varphi(w), w v)-$ $v$, at fixed $v$.

Such an excess demand function cannot be anything (work in progress).

Again the income effects associated with the above term $+\sum_{k} F(0) \sum_{h}\left(\partial D_{h} / \partial R\right)\left(v_{0, h}^{k}-\right.$ $\left.F^{h} x_{0, h}\right) d w_{k}$ play a crucial role in the analysis. Conditions for the existence of a Keynes effect (decrease in wages involves increase in unemployment) are displayed (work in progress).

\section{The standard theory of trade : comparative statics.}

Stylised model and questions. We have already argued that our analyis provide a more general versions perspective on the findings associated with the StoplerSamuelson or Ribzcinsky'theorems, as well as possibly additional insights on what is the counterpart of non-intensity reversal. Let us sketch some comparative statics analysis of a simple trade model with only two countries.

Let us consider two countries that have the same production sector, hence the same $\varphi(w)$. Assume that they have different vector endowments $V^{a}, V^{b}$, and for the sake of simplicity, representative consumers $a, b$ that, when needed for the simplicity of the argument, may have the same homothetic preferences (so that there is a representative consumer for the world).

The object of theoretical interest is the graph of the trade equilibrium correspondence : how do vary equilibrium (ia) with the data? Here is a limited sample of questions.

Q1- for a given set of economies, does there exists a non specialized trade equilibrium, where all goods are produced at the same price in every country, (which would then coincide with the world equilibrim with factor mobility), does there exist several and how many ? how do they vary with the data and how do we switch to specialisation ? 
Q2 - In a non specialized trade equilibrium, are the price of factors equalized across countries ? When is it the case in a specialized trade equilibrium ?

Q3 - Can specialized equilibria coexist in a given world with non specialized trade equilibria. etc..

Some answers.. We claim that the above analysis, or its suggested extension, provides appropriate tools for a systematic investigation of the above questions. Again, it is difficult to go very far into the analysis without understanding better the graph of the Walras correspondence of an isolated economy.

Together with the other tools presented here, the factor price equalisation results shed light on the graphof the trade equilibrium correspondence.

-When enough goods are traded (more than $2 K$ ), then factor price equalisation obtains. Or, similarly, absence of factor price equalisation involves important specialisation and limited trade.

- With less traded goods, $K<L<2 K$, the critical economies where factor price equalisation does not hold provide interesting information on the graph of the trade correspondence and possibly on the coexistence of specialized and non-specialized equilibria.

\section{Others.}

Production theory : extensions, joint production. Also, the results also have applications to the case of joint production, a context in which the line of approach of Bidard (1990) provides a natural entry.

Non standard trade theories.. In case where prices are not competitive prices but result from oligopolistic pricing, as in the new trade theory, prices are still related ot marginal costs and then the connections prices of goods- factor prices are not basically dissimilar with what has been established here.

Taxation. Taxation theory -either from the viewpoint of reform or of the description of the manifold of equilibria has been developed oftne under the assumption of decreasing returns and not of constant returns.

Intertemporal production theory.. We believe that our results provide starting points for an improved investigation of several other subjects, such as the analysis of observable consequences of general equilibrium theory, in the line of previous attempts in the framework of an exchange economy, as in Brown-Matzkin (1996) or Chiappori-Ekeland-Kubler-Polemarchakis (2000) or the intertemporal theory of production, rather than the atemporal one considered here. In an intertemporal context where capital depreciate within the period, goods at period $t$ are produced from goods produced at period $t-1$ and from factors available at period $t$. Most of our results 
apply and allow to derive dynamic non-substitution theorems as well as the intertemporal prices of goods as a function of the time varying relative prices of factors and of inter-period interest rates. And for exemple, the deformation of prices due technical progress can be assessed. When capital does not depreciate immediately, some additional work is required to adapt the present results.

\section{Conclusion.}

We first provide a final assesment of our results, in order to make clearer their position in the complex literature to which they add.

The results of Section 2 to 4 apply whatever the structural assumption on the production system (producing good $l$ may require all or a subset of goods and factors, but this is given at the outset). Our assessment of the geometry of the global production set in Section 3 is claimed to be original, although some of its aspects have been understood and presented in special contexts in the case where goods are produced from factors only. The algebraic perspective of Section 4 provides a generalisation to the case where goods are produced from factors and goods of results known in the case where goods were produced from factors only.

The results of Section 5 are novel, to the best of our knowledge. The genericity argument has been presented in the case where all other goods are needed in the production of any good, but it has counterparts under different structural assumptions.

Let us make some final comments on our own motivation for this this research.

Although "general equilibrium", as a subject aiming at maximal generality in the understanding of the systemic aspects of economic interactions, is no longer very active, its intellectual apparatus is extremely alive in many specific fields where it has been either partly adapted (standard or new trade theories) and/or simplified to the extreme (with a representative consumer). Also a lot of influential work on policy analysis rests on the use of computable general equilibrium models that adopt specific modelling options that both allow to simplify the general analysis and make it empirically plausible.

In all cases, it may be argued that an equilibrium theory of intermediate generality is needeed, not mainly to provide provide specific applications of the intellectual products of the most abstract theory, but essentially and crucially to improve upon the present specific models and increase their policy relevance.

The present paper is an attempt of going further into a theory of production of intermediate generality, aiming at hte just described objectives. As argued above, the most systematic attempts in this direction have been made in international trade theory untill the eighties; however, the results presented here are neither uniquely motivated by trade, nor primarily applicable to this field. They concern a broader research program, and aim at providing some useful harbour to additional investigation 
in this program.

\section{BiBLIOGRAPHY}

Abraham R., and Robbin J. 1967. "Transversal mappings and flows." Benjamin . Arrow K., and F. Hahn. 1971. "General Competitive Analysis." Holden Day . Aubin, J.P., and I. Ekeland. 1984. "Applied nonlinear analysis." Wiley-Interscience. Bidard, C. 1990. "An algorithmic theory of the choice of techniques." Econometrica 58, 4:839-859.

Brown, D. and R.Matzkin. 1996. "Testable restrictions on the Equilibrium manifold", Econometrica, 1249-1262

Chiappori P.A, Ekeland I, Kubler E, Polemarchakis H. (2000) "The identification of preferences from equilibrium prices", Journal of Economic Theory, 102 (2002), p. 403-420

Dixit, A., and V. Norman. 1980. "Theory of international trade." James Nisbet and Cambridge University Press, Digswell Place .

Ethier, W.J. 1984. "Higher dimensional issues in trade theory." Handbook of international economics 1:131-184.

Findlay R, (1995) "Factor proportions, trade and growth", MIT Press

Freeman, R, 1995, "Are our wages set in Beijing ?". The Journal of Economic Perspectives, 9-3: 15-32.

Gale, D., and H. Nikaido. 1965. "The Jacobian matrix and the global univalence of mappings." Mathematische Annalen 159: 81-93.

Hildenbrand, W. 1994. "Market demand." Princeton University Press .

Horn, R., and C. Johnson. 1991. "Topics in matrix analysis." Cambridge University Press.

Jones, R., and J. Scheinkman. 1977. "The relevance of the two sector production model in trade theory." Journal of Political Economy 85:909-935.

Kehoe T, 1983, "Regularity and Index Theory for Economies with Smooth Production Technologies", Econometrica, 51, 4, 895-918.

Mac Kenzie, L. 1955. "Equality of factor prices in world prices." Econometrica 23:239-257.

Malinvaud, E. 1967. "Decentralised Procedures for Planning." in Activity Analysis and the theory of Growth and Planning, edited by Bacharach eds. New York.

Mas Colell, A. 1979a. "Homeomorphisms of compact convex sets and the Jacobian matrix." SIAM journal of mathematical Analysis 10:1105-1109.

Mas Colell, A. 1979b. "Two propositions on the global univalence of costs functions." in General equilibrium, growth and trade, edited by J.R Green and J.A Scheinkman. New York: Academic Press.

Mas Colell, A. 1991, "On the uniqueness of equilibrium once again", Chapter 12 in "Equilibrium theory and Applications", edited by W. Barnett, B. Cornet, 
C.d'Aspremont, J; Gabzewicz and A. Mas Colell. Cambridge, UK, Cambridge University Press.

Mas-Colell, A., M. Whinston, and J.R. Green. 1995. "Microeconomic theory." in Oxford University Press. New York.

Neary, P. 1985. "Two by two international trade theory with many goods and factors." 53,5:1233-1247.

O'Rourke, Kevin H, Taylor, Alan M and Williamson, Jeffrey G, 1996. "Factor Price Convergence in the Late Nineteenth Century," International Economic Review, vol. 37(3), pages 499-530.

O’Rourke, Kevin H and Williamson, Jeffrey G, 2000. "The Heckscher-Ohlin Model Between 1400 and 2000: When It Explained Factor Price Convergence, When It Did Not, and Why," CEPR Discussion Papers 2372,

Samuelson, P. 1953. "Prices of factors and goods in general equilibrium." Review of Economics Studies 21,1,:1-20.

Shafer, W., and H. Sonnenschein. 1982. "Market Demand and Excess Demand Functions." in Handbook of Mathematical Economics, edited by Kenneth Arrow and Michael Intriligator. Amsterdam: North Holland.

Sraffa, P. 1960. Production of comodities by means of commodities. Cambridge.

Travis W. 1972, "Production, Trade and Protection when there are Many Commodities and two Factors", American Economic Review, 87-106.

Woodland, A. 1983. "International trade and resource allocation." , edited by North Holland. Amsterdam.

\section{APPENDiX}

\subsection{Appendix 1 : A detailed primal view of the problem..}

Definition 1. Given a production plan $y \in Y$, any family $y_{\ell}, 1 \leq \ell \leq L$, with $y_{\ell}=\left(x_{\ell}, v_{\ell}\right) \in Y_{\ell}$ and $y=\sum y_{\ell}$ will be called a $y$-allocation. A production plan $y$ is non-specialized if there exists a $y$-allocation with $x_{\ell}^{\ell}>0$ for all $\ell$. The set of all non-specialized production plans will be denoted by $Y_{N S}$ :

$$
Y_{N S}=\left\{\sum y_{\ell} \mid y_{\ell}=\left(x_{\ell}, v_{\ell}\right) \in Y_{\ell}, x_{\ell}^{\ell}>0 \forall \ell\right\}
$$

In a non specialized production plan, gross production $x_{\ell}^{\ell}$ of every good $\ell$ is positive, but not necessarily net production $x^{\ell}=\sum_{k} x_{k}^{\ell}$. The case when $x_{\ell}^{\ell}=0$ for some good $\ell$ is the case when production is specialized, in the terminology of international trade.

$>$ From now on, we shall write NS instead of non-specialized. Take a NS production plan $y=\sum y_{\ell}$, with $y_{\ell}=\left(x_{\ell}, v_{\ell}\right) \in Y_{\ell}$. We have: 


$$
\begin{aligned}
v^{k} & =\sum_{\ell} v_{\ell}^{k}, \quad v_{\ell}^{k}<0 \quad \forall \ell \\
x^{i} & =\sum_{l} x_{\ell}^{i}, \quad x_{\ell}^{\ell}>0 \quad \forall \ell \\
x_{\ell}^{\ell} & \leq f_{\ell}\left(-x_{\ell}^{-\ell},-v_{\ell}\right), \quad \forall \ell
\end{aligned}
$$

Since we have constant returns to scale, the set $Y_{N S}$ is a convex cone. It makes sense to normalize the production plans in each sector by considering the inputs required to produce one unit of good. Set:

$$
Y_{\ell}^{1}=\left\{y=(x, v) \in Y_{\ell} \mid x_{\ell}^{\ell}=1\right\}
$$

so that any vector $y \in Y_{\ell}^{1}$ describes one of the many ways to produce one unit of $\operatorname{good} \ell$.

Definition 2. Let $y$ be a NS production plan. A family $\left(\widehat{y}_{\ell}, \lambda_{\ell}\right), 1 \leq \ell \leq L$ will be called a normalized $y$-allocation if:

$$
\begin{aligned}
\widehat{y}_{\ell} & =\left(\hat{x}_{\ell}, \hat{v}_{\ell}\right) \in Y_{\ell}^{1} \\
\lambda_{\ell} & >0 \forall \ell \\
y & =\sum_{\ell} \lambda_{\ell} \widehat{y}_{\ell}
\end{aligned}
$$

Equation (19) is the normalization condition. It is clear from the definitions that for any NS production plan, as defined by equations (16), (17), and (18), there is a corresponding normalized allocation.

The economic interpretation is straightforward. The $\left(-\hat{x}_{\ell}^{-\ell},-\hat{v}_{\ell}\right)$ are the inputs required to produce one unit of good $\ell$, with the chosen technology, and the numbers $\lambda_{\ell}$ then denote the level at which this technology has to be set in order to produce (jointly with the others) the bundle $y$.

To stress this interpretation, and make the connection with the classical Leontieff model, let us introduce new notations. Set:

$$
\begin{aligned}
& a_{\ell}^{i}(y)=-\widehat{x}_{\ell}^{i}>0 \quad \forall i \neq \ell \\
& a_{\ell}^{\ell}(y)=0 \\
& b_{\ell}^{k}(y)=-\widehat{v}_{\ell}^{k}>0 \quad \forall k
\end{aligned}
$$

Equations (18) and (21) can then be written : 


$$
\begin{aligned}
1 & \leq f_{\ell}\left(a_{\ell}, b_{\ell}\right) \\
x^{i} & =\lambda_{i}-\sum_{\ell} a_{\ell}^{i} \lambda_{\ell} \\
v^{k} & =-\sum_{\ell} b_{\ell}^{k} \lambda_{\ell}
\end{aligned}
$$

or, with straigthforward notation :

$$
x=(I-A) \lambda, v=-B \lambda,
$$

where $^{22} A=\left(a_{\ell}^{i}\right)$ and $B=\left(b_{\ell}^{k}\right)$ are respectively $L \times L$ and $K \times L$ matrices with positive coefficients (except for the diagonal of $A$ which is zero), the $\ell$-th column of which consists of the input vector, respectively in goods and factors, used for the production of one unit of good $\ell$ in the chosen allocation.

9.2. Appendix 2 : Efficiency from the dual viewpoint. The next lemma summarizes our conclusions with the further implications that obtain when the efficient production plan $y$ is non-specialized ${ }^{23}$ :

Lemma 29. If a NS-production plan $y$ is efficient, then there exists a unique remuneration plan, $q \in E_{1}$ such that :

$$
q^{\prime} y_{\ell}=\operatorname{Max}\left\{q^{\prime} y \mid y \in Y_{\ell}\right\}=0 \quad \forall \ell
$$

and there is a unique normalized $y$-allocation $\left(\widehat{y}_{\ell}, \lambda_{\ell}\right)$. The $\widehat{y}_{\ell}, 1 \leq \ell \leq L$, are linearly independent, and $q^{\prime} \widehat{y}_{\ell}=0$ for all $\ell$.

This lemma enables us to define maps $\gamma_{\ell}, \lambda_{\ell}$ and $\pi_{1}$ from $Y_{N S E}$ to $Y_{\ell}^{1}, R_{++}$and $E_{1}$ by:

$$
\begin{aligned}
\gamma_{\ell}(y) & =\widehat{y}_{\ell} \\
\lambda_{\ell}(y) & =\lambda_{\ell} \\
\pi(y) & =q
\end{aligned}
$$

In other words, $\gamma_{\ell}(y)$ is the unique (and efficient) way of producing one unit of good $\ell$ when the global production plan $y=(x, v)$ is aimed for. The activity level

\footnotetext{
${ }^{22}$ In a Leontieff economy, with strict complementarities, $A$ and $B$ are given a priori, while here they arise endogenously, but not necessarily uniquely.

${ }^{23}$ The linear independence of the $\widehat{y}_{\ell}$ follows from the fact that only technology $\ell$ can produce good $\ell$. The remaining follows straightforwardly from what we just said.
} 
at which technology $\ell$ has to operate, using this production plan, is specified by $\lambda_{\ell}(y)>0$, so that $y=\sum_{\ell} \lambda_{\ell}(y) \gamma_{\ell}(y)$. The normalized remuneration plan supporting this production plan is $\pi(y)$, with $\pi(y)^{\prime} y=0$.

We shall also consider later on the map:

$$
\gamma: Y_{N S E} \longrightarrow Y_{1}^{1} \times \ldots \times Y_{L}^{1}
$$

defined by $\gamma=\left(\gamma_{1}, \ldots, \gamma_{L}\right)$

\section{APPENDIX 3 : THE GEOMETRY.}

$C(y)$ is an $L$-dimensional polyhedral cone $^{24}$ in $R^{K+L}$, with vertex at 0 , and edges carried by the $\gamma_{\ell}(y)$. We know that $y \in C(y)$ (just take $\mu_{\ell}=\lambda_{\ell}(y)$ ). Because of formula (1), we see that $T(\bar{y})=\{y \mid \pi(\bar{y}) y=0\}$ is the tangent hyperplane to $Y$ at $\bar{y}$.

Proposition 30. $C(y)=T(y) \cap Y_{N S E}$

Proof. Consider the closure of $C(y)$ :

$$
\bar{C}(y)=\left\{\sum_{\ell} \mu_{\ell} \gamma_{\ell}(y) \mid \mu_{\ell} \geq 0,1 \leq \ell \leq L,\right\}
$$

It is a closed convex cone, containing each of the $\gamma_{\ell}(y)$. Because of equation (??), each of the $\gamma_{\ell}(y)$ also belongs to the hyperplane $T(y)$. It follows immediately that $\bar{C}(y) \subset T(y)$. As a consequence of our constant returns to scale assumption, all of $\bar{C}(y)$ is contained in the global production set $Y$. Hence $\bar{C}(y) \subset T(y) \cap Y$, and $C(y) \subset T(y) \cap Y_{N S E}$.

Conversely, if $z \in T(y) \cap Y_{N S E}$, then there is a normalized $z$-allocation $\left(\gamma_{\ell}(z), \lambda_{\ell}(z)\right), 1 \leq$ $\ell \leq L$. By equation (??), each $\gamma_{\ell}(z)$ maximizes $\pi(y)^{\prime} z_{\ell}$ subject to $z_{\ell} \in Y_{\ell}$, and it follows from uniqueness that $\gamma_{\ell}(z)=\gamma_{\ell}(y)$ and $z \in C(y)$. Hence $T(y) \cap Y_{N S E} \subset C(y)$

As a consequence of Proposition 3, we have that

$$
Y_{N S E}=\cup_{y \in Y_{N S E}} C(y)
$$

so that the cone $Y_{N S E}$ is a disjoint (possibly infinite) union of $L$-dimensional polyhedral cones.

Theorem 31. Every $x \in Y_{N S E}(v)$ belongs to a single facet Facet $(x) \subset Y_{N S E}(v)$. If $L \geq K$, this facet has dimension $(L-K)$, and $x$ belong to its relative interior

\footnotetext{
${ }^{24}$ In the case $K=1, L=2$ we investigated in Section ??, $G(y)$ is a two-dimensional cone in $\left(x_{1}, x_{2}, v\right)$-space. Its section by the plane $v=1$ is the line segment we depicted in Figure 1.
} 
Proof. If $y=(x, \bar{v}) \in Y_{N S E}$, denote by $T(y)$ the tangent hyperplane (in $R^{K+L}$ ) to $Y_{N S E}$ at $y$ and by $T(x)$ the tangent hyperplane (in $\left.R^{L}\right)$ to $Y_{N S}(\bar{v})$ at $x$. Since $Y_{N S E}(\bar{v})$ is the intersection of $Y_{N S E}$ with $R^{L} \times\{v\}$, we must have:

$$
T(x)=T(y) \cap\left(R^{L} \times\{v\}\right)
$$

By definition:

$$
\operatorname{Facet}(x)=Y_{N S E}(\bar{v}) \cap T(x)=Y_{N S E}(\bar{v}) \cap T(y) \cap\left(R^{L} \times\{v\}\right)
$$

and using proposition 3 we find that

$$
\operatorname{Facet}(x)=C(y) \cap\left(R^{L} \times\{v\}\right)
$$

In $R^{L+K}, C(y)$ is an $L$-dimensional cone and $R^{L} \times\{v\}$ an $L$-dimensional affine subspace transversal to the cone. Their intersection is an $(L-K)$-dimensional cone, as announced.

There are two limiting situations, $K=1$ (one factor) and $K=L-1$ (many factors). In the first case, we get the classical non-substitution theorem of Arrow and Samuelson

10.1. Appendix 4 : Primal and dual.. The next result summarizes our findings up to now, while explicitly relating the dual notation of this subsection with the primal notation of the previous one. It basically tells us about the decentralization role of remuneration plans.

Proposition 32 [Decentralization]. If $y=(x, v)$ is an efficient NS production plan, then :

1. There is a unique normalized y-allocation $\left(\gamma_{\ell}(y), \lambda_{\ell}(y)\right), 1 \leq \ell \leq L$, with $\lambda_{\ell}(y)>0$, and a unique normalized remuneration plan $\pi(y)=(p(y), w(y))$ supporting $y$

2. If $q=(p, w)=\pi(y)$, so that $p_{1}=1$, the matrices $A(q)$ and $B(q)$ obtain from the $\gamma_{\ell}(y)$ as follows:

$$
\gamma_{\ell}(y)=\left(-a_{\ell}^{1}(q), \ldots,-a_{\ell}^{\ell-1}(q) \quad 1-a_{\ell}^{\ell+1}(q), \ldots,-a_{\ell}^{l}(q),-b_{\ell}^{1}(q), \ldots,-b_{\ell}^{K}(q)\right)
$$

3. The matrices $A(q)$ and $B(q)$ are positively homogeneous of degree 0 , and we have

$$
p(y)=w B(q)(I-A(q))^{-1}
$$


4. We have:

$$
\begin{aligned}
\lambda(y) & =(I-A(q))^{-1} x \\
-v & =B(q) \lambda(y)=B(q)(I-A(q))^{-1} x
\end{aligned}
$$

The converse of Proposition ?? is the following :

Proposition 33. Suppose $q=(p, w) \in R_{++}^{L+K}$ is such that $p=w B(q)(I-A(q))^{-1}$. For any $\lambda \in R_{++}^{L}$, define $y_{\lambda}=\left(x_{\lambda}, v_{\lambda}\right)$ by

$$
\begin{aligned}
& x_{\lambda}=(I-A(q)) \lambda \\
& v_{\lambda}=-B(q) \lambda
\end{aligned}
$$

Then $y_{\lambda}$ is in $Y_{N S E}$, and $q=a \pi\left(y_{\lambda}\right)$ for some $a>0$.

Proof. If the above condition holds for some $q=(p, w)>0$, the $\ell$-th columns of the matrices $A(q)$ and $B(q)$ define a cost minimising bundle for the production of one unit of good $\ell$, and then, for each $\ell$, a profit maximising $y_{\ell} \in Y_{l}$ (with zero profit). This implies that for any $\lambda \in R_{++}^{L}$, the production plan $y_{\lambda}$ defined by (??) and (??) is the sum of profit maximising bundles; therefore it is efficient and belongs to $Y_{N S E}$. The uniqueness of (the direction of) $q$ follows from the same argument as above

10.2. Appendix 5 :Proof of the Preparation Theorem 14.. We begin by proving that the projection map $\psi$ from $\Sigma$ to $R_{++}^{K}$ is one-to-one.

Lemma 34. If $\left(p^{1}, w\right)$ and $\left(p^{2}, w\right) \in \Sigma$, then $p_{1}=p_{2}$

Proof. Suppose otherwise, so that $p_{1} \neq p_{2}$. Introducing the cost functions, as in equation (7) we have $p_{1}=c\left(p_{1}, w\right)$ and $p_{2}=c\left(p_{2}, w\right)$. Consider

$$
t=\operatorname{Max}_{\ell}\left(p_{\ell}^{1} / p_{\ell}^{2}\right)
$$

and assume without loss of generality that $t>1$. Set $p^{3}=t p^{2}$, so that $p_{\ell}^{3} \geq p_{\ell}^{1} \forall \ell$, and $p_{k}^{3}=p_{k}^{1}$ for some $k$. But $t p^{2}=c\left(t p^{2}, t w\right)$ by homogeneity, and hence, using the fact that the cost is a decreasing function of prices:

$$
p_{\ell}^{3}=c_{\ell}\left(p^{3}, t w\right)>c_{\ell}\left(p^{1}, w\right)=p_{\ell}^{1} \forall \ell
$$

Hence $p_{k}^{3}=p_{k}^{1}$, a contradiction.

We then prove that the projection map $\psi$ from $\Sigma$ to $R_{++}^{K}$ covers all of $R_{++}^{K}$. We begin with a technical result. By $\mathrm{C} 4$, there is some $y \in Y_{N S E} \cap\left(R_{++}^{L} \times R^{K}\right) \subset Y_{N S E}^{++}$. Take a supporting price vector, for instance $q=\pi(y)$, and set $A=A(q)$ and $B=B(q))$ 
Lemma 35. Take $q^{0}=\left(p^{0}, w^{0}\right) \in \Sigma$. Then, $p^{0} \leq B^{\prime} w^{0}\left(I-A^{\prime}\right)^{-1}$

Proof. Consider the goods price vector $p^{1}$ defined by $p^{1}=B^{\prime} w^{0}\left(I-A^{\prime}\right)^{-1}$. We have $p^{1}=B^{\prime} w^{0}+A^{\prime} p^{1}$, meaning that the vector $p^{1}$ gives the unitary costs of production if the input prices are $\left(p^{1}, w^{0}\right)$ and the plan $y$ is used. Set $p^{2}=$ $B\left(p^{1}, w^{0}\right)^{\prime} w^{0}+A\left(p^{1}, w^{0}\right)^{\prime} p^{1}$. The vector $p^{2}$ gives the unitary costs of production if the prices are $\left(p^{1}, w^{0}\right)$ and a cost-minimizing plan is used; these costs must be less than the costs of production using any other plan, so that $p^{2} \leq p^{1}$.

The algorithm then proceeds : set $p^{3}=B\left(p^{2}, w^{0}\right)^{\prime} w^{0}+A\left(p^{2}, w^{0}\right)^{\prime} p^{2}$; then $p^{3} \leq p^{2}$, and so on. The sequence $p^{n}$ is a decreasing sequence of positive vectors, so that it must converge to some $p$ satisfying $p=\left(w^{0}\right)^{\prime} B\left(p, w_{0}\right)+p^{\prime} A\left(p, w^{0}\right)$, and by Lemma ?? we must have $p=p^{0}$. We have proved that $p^{1} \geq p^{2} \geq \ldots \geq p=p^{0}$.

Lemma 36. The projection map $\psi$ from $\Sigma$ to $R_{++}^{K}$ covers all of $R_{++}^{K}$

Proof. We shall prove that the image $\psi(\Sigma)$ is open and closed in $R_{++}^{K}$. Openness follows from lemma 13. To show closedness, consider a sequence $w^{n}$ in $\psi(\Sigma) \cap R_{++}^{K}$, converging to some $w \in R_{++}^{K}$. Take the corresponding sequence $p^{n}$ such that $\left(p^{n}, w^{n}\right) \in \Sigma$, so that $w^{n}=\psi\left(p^{n}, w^{n}\right)$. By the preceding lemma, the $p^{n}$ are uniformly bounded, so there is a convergent subsequence to some $p$, and $p^{\prime}=w^{\prime} B(p, w)(I-A(p, w))^{-1}$ by continuity. Since $w \in R_{++}^{K}$ and the matrices $B$ and $(I-A)^{-1}$ have positive coefficients, we must have $p \in R_{++}^{L}$. So the image $\psi(\Sigma)$ is closed in $R_{++}^{K}$. Since $R_{++}^{K}$ is connected, the only subsets which are both open and closed are the empty set $\varnothing$ and $R_{++}^{K}$ itself. The result follows.

The map $\psi$ can be inverted on $R_{++}^{K}$, yielding the Preparation Theorem.

\section{Appendix 6 : Genericity.}

Consider a property $P(\theta)$, depending on $\theta \in \Theta$. In our context, $\theta$ indexes parameters that generate different specifications of a model. We would like a suitable mathematical notion to translate the idea that $P$ holds true "in general", that is, for "most" specifications of the model.

The standard way to make this idea precise is to be Bayesian. In this approach, one endows the parameter space $\Theta$ with a positive measure $\mu$, and says that a property $P$ holds "in general" if it holds $\mu$-almost surely, that is, if:

$$
\mu[\Theta-\Omega]=0 .
$$

where $\Omega$ is the set of $\theta \in \Theta$ such that $P(\theta)$ is true. Taking $\Theta$ to be the real line, $\mu$ to be the Lebesgue measure, and $P(\theta)$ to be the property " $\theta$ is irrational", we will thus state (correctly) that real numbers are irrational in general 
The problem with this approach lies in choosing the measure $\mu$. In the absence of a priori information, the Lebesgue measure may seem an appropriate starting point, because it is translation-invariant. Unfortunately, it is not a probability, so that it will not fit easily into a Bayesian framework. One may overlook this problem by pointing out that it is not the measure $\mu$ which is important, only the $\mu$-negligible subsets are. One then runs into a second problem, namely the fact that there is no equivalent of Lebesgue measure in infinite-dimensional spaces, such as function spaces. On such spaces, there is no translation-invariant measure that one could use to define negligible sets without a priori information.

One then has to choose a probability. If $\Theta$ is finite-dimensional, one runs into the familiar problems of Bayesian theory. There is no reason why two different observers should share the same prior, or even why they should have priors which are absolutely continuous with respect to each other. If $\Theta$ is infinite-dimensional, there is the added difficulty that there are very few probabilities on such spaces; we have already noted that there is no equivalent of the Lebesgue measure. It will thus be very difficult to give a mathematically precise and generally accepted meaning to the statement "continous functions are not linear in general".

In this paper, we will use a second approach, which is due to Rene Thom; see Abraham and Robbin (1967) and Aubin and Ekeland (1984) for discussions of this approach. It consists in endowing the parameter space $\Theta$, not with a positive measure, but with a complete metric, so that all Cauchy sequences converge. This approach defines a property $P(\theta)$ to be generic if there is a sequence of subsets $U_{n} \subset \Theta$ such that

$$
\begin{aligned}
& U_{n} \text { is open and dense } \forall n \\
& \Omega \supset \cap_{n} U_{n}
\end{aligned}
$$

where $\Omega$ is again the set of $\theta$ where $P(\theta)$ holds true.

As an example, the property of being irrational is generic among real numbers. Indeed, the set of rational numbers is countable, so the set of irrational numbers is the intersection of a countable number of open dense sets. Let $\rho_{n}, n \in N$, be the rationals, then the set of irrationals will be $\cap_{n} U_{n}$, where $U_{n}$ is the complement of $\left\{\rho_{n}\right\}$. This approach also works in our second example. Denote the space of continuous functions on $[0,1]$ by $C^{0}([0,1])$, and the subset of affine functions by $A([0,1]) . A([0,1])$ is closed and has empty interior, so that its complement is an open dense subset. So, nonlinearity is a generic property in $C^{0}([0,1])$.

If two properties $P_{1}$ and $P_{2}$ are generic, so are $P_{1} \wedge P_{2}\left(P_{1}\right.$ and $\left.P_{2}\right)$ and $P_{1} \wedge P_{2}\left(P_{1}\right.$ or $P_{2}$ )As a consequence, if $P$ is generic, then its negation not $P$ cannot be generic. More generally, if a sequence of properties $P_{n}$ are all generic, then so is $\wedge_{n} P_{n}$. In other words, generic properties behave in the same way as properties that are true almost surely, although there is no underlying measure to support them. 
Thom's approach has the great advantage that in many cases of interest there is a natural metric on the parameter space $\Theta$, even if there is no natural probability measure on it. So people will agree on what is generic or not, although they do not agree on which sets have measure zero.

\section{Proof of Theorems ?? and ?? .}

Defining appropriate sets and maps. Let us denote by $\mathcal{F}^{r}$ the set of all $f=\left(f_{1}, \ldots, f_{L}\right)$ which satisfies conditions $\mathrm{C} 1$ to $\mathrm{C} 4$, each $f_{\ell}$ being $r$ times continously differentiable. Note that there is no natural norm on this space (because our assumptions allow $f_{\ell}$ or its derivatives to become unbounded when its arguments go to zero or infinity), but nevertheless there is a natural metric, the precise description of which is given in what follows. In the statements of Theorems ?? and ??, genericity is understood with respect to $\mathcal{F}^{r}$, for some $r \geq 2$.

Assume $L>K$. Set $J=L+1-K$ and denote by $\mathcal{C}^{r}$ the space of all real-valued functions $f$ on $R_{++}^{J}$ which are positively homogeneous of degree one, $r$ times continuously differentiable on $R_{++}^{J}$, and vanish on the boundary. Let $S=\left\{z \in R_{++}^{J} \mid \sum z_{j}<1\right\}$ be the standard simplex in $R^{J}$. Choose a family $\Omega_{n}$ of open subsets of $S$ such that $\overline{\Omega_{n}} \subset \Omega_{n+1} \subset S$ and $S=\cup \Omega_{n}$. Endow $\mathcal{C}^{r}$ with the following family of semi-norms:

$$
\begin{aligned}
q^{0}(f) & =\max \{|f(z)| \mid z \in S\} \\
q_{i_{1} \ldots i_{K}}^{n}(f) & =\max \left\{\left|\frac{\partial^{m} f}{\partial^{i_{1}} z_{1} \ldots \partial^{i_{J}} z_{J}}(w)\right| \mid w \in \Omega_{n}\right\}
\end{aligned}
$$

where $1 \leq m \leq r, i_{1}+\ldots+i_{J}=m$. This is a countable family of semi-norms, which we relabel simply as $\left\{q_{k}\right\}_{k \geq 1}$. It is well known that the distance

$$
d(f, g)=\sum_{k} \frac{1}{k^{2}} \max \left\{q_{k}(f-g), 1\right\}
$$

turns $\mathcal{C}^{r}$ into a separable and complete metric space. To say that $f_{n} \rightarrow f$ in $\mathcal{C}^{r}$ means that $f_{n}$ converges to $f$ uniformly on $S$ (and hence uniformly on compact subsets of $R_{+}^{J}$, including the boundary) and that all partial derivatives of $f_{n}$ converge to the corresponding derivatives of $f$, uniformly on compact subsets of $S$ (and hence uniformly on compact subsets of $R_{++}^{J}$, excluding the boundary). Every neighbourhood of $f$ in $\mathcal{C}^{r}$ contains a neighbourhood of the form $\left\{g \mid q_{k}(f-g) \leq \varepsilon\right\}$ for some $k$ and $\varepsilon$.

Let us denote by $\mathcal{F}$ the set of all $f=\left(f_{1}, \ldots, f_{L}\right)$ which satisfies conditions (1) to (4). Set $\mathcal{F}^{r}=\mathcal{F} \cap \mathcal{C}^{r}$, and endow $\mathcal{F}^{r}$ with the induced topology. Since $r \geq 2, \mathcal{F}^{r}$ is an open subset of $\mathcal{C}^{r}$, so that $\mathcal{F}^{r}$ is a Baire space.

In the above, we have defined maps $A: R_{++}^{K+L} \rightarrow \mathcal{L}\left(R^{L}, R^{L}\right), B: R_{++}^{K+L} \rightarrow$ $\mathcal{L}\left(R^{K}, R^{L}\right)$ (see section 4.1) and $\varphi: R_{++}^{K} \rightarrow R_{++}^{L}$ (see Theorem 14). These maps 
depend on $f \in \mathcal{F}$, and we shall henceforth write them $A_{f}, B_{f}$ and $\varphi_{f}$ to stress this dependence. Note that they are all positively homogeneous, $A$ and $B$ of degree 0 , and $\varphi_{f}$ of degree one.

For the purposes of the proof, it will be convenient to normalize prices by setting $w_{1}=1$. We set:

$$
W_{1}=\left\{w \in R_{++}^{K} \mid w_{1}=1\right\}
$$

Consider the $(2 K-1)$-dimensional space:

$$
\Delta=\left\{\left(w^{1}, w^{2}, \lambda\right) \in W_{1} \times W_{1} \times R_{++} \mid w^{1} \neq w^{2}\right\}
$$

and the map $\Psi: \mathcal{F}^{r} \times D \rightarrow R^{L}$ defined by

$$
\Psi\left(f, w^{1}, w^{2}, \lambda\right)=\lambda \varphi_{f}\left(w^{1}\right)-\varphi_{f}\left(w^{2}\right)
$$

\section{Transversality..}

Proposition 37. Generically in $\mathcal{F}^{r}$, the partial map $\Psi_{f}: D \rightarrow R^{L}$ defined by $\Psi_{f}\left(w^{1}, w^{2}, \lambda\right)=\Psi\left(f, w^{1}, w^{2}, \lambda\right)$ is transversal to the origin in $R^{L}$.

Again, we refer to Abraham and Robbin (1967) and to Aubin and Ekeland (1984) for a definition of transversality and a statement of the Thom transversality theorem. Saying that $\Psi_{f}$ is transversal to the origin means that either $\Psi_{f}^{-1}(0)$ is empty, or that at every $\left(w^{1}, w^{2}, \lambda\right) \in \Psi_{f}^{-1}(0)$, the tangent map $D \Psi_{f}$ is onto. Theorems ?? and ?? both will follow from this proposition.

We shall first prove a weaker result. Fix a compact subset $C \subset W_{1}$, set:

$$
\Delta^{C}=\left\{\left(w^{1}, w^{2}, \lambda\right) \in C \times C \times R_{++} \mid w^{1} \neq w^{2}\right\}
$$

and denote by $\Psi_{f}^{C}$ the restriction of $\Psi_{f}$ to $\Delta^{C}$.

Lemma 38. For every compact subset $C \subset W_{1}$, every $f \in \mathcal{F}^{r}$ has an open neighbourhood $\mathcal{N}(f)$ such that, generically with respect to $g \in \mathcal{N}(f)$, the partial map $\Psi_{g}^{C}: \Delta^{C} \rightarrow R^{L}$ is transversal to the origin in $R^{L}$.

Proof. Let $C$ and $f$ be given. Let $G_{f}=\left\{\left(\varphi_{f}(w), w\right) \mid w \in C\right\}$ be the graph of $\varphi_{f}$ over $C$; it is a compact subset of $R_{++}^{L+K-1}$. Take a bounded open subset $\mathcal{V} \subset R_{++}^{L+K-1}$ such that $\overline{\mathcal{V}} \subset R_{++}^{L+K-1}$ and $G_{f} \subset \mathcal{V}$. For each $q=(p, w)$ in $G_{f}$, the matrices $A_{f}(q)=$ $\left(a_{\ell}^{i}\right)$ and $B_{f}(q)=\left(b_{\ell}^{k}\right)$ are obtained by solving for every $\ell$ the following equations in $a_{\ell} \in R_{++}^{L}, b_{\ell} \in R_{++}^{K}$ and $\lambda_{\ell}>0$ :

$$
\begin{aligned}
a_{\ell}^{\ell} & =0 \\
D f_{\ell}\left(a_{-\ell}, b_{\ell}\right) & =\lambda_{\ell} q \\
f_{\ell}\left(a_{-\ell}, b_{\ell}\right) & =1
\end{aligned}
$$


which are the optimality conditions in problem (5). Applying the implicit function theorem, with the help of condition 3 , we find that the solution $\left(a_{-\ell}, b_{\ell}\right)$ and the Lagrange multiplier $\lambda_{\ell}$ depend smoothly on $q$. It follows that there is a bounded open subset $\mathcal{U}_{\ell}$ the closure of which, $\overline{\mathcal{U}}_{\ell}$, is contained in $R_{++}^{K+L-1}$, such that $\left(A_{f}(q), B_{f}(q)\right) \in$ $\mathcal{U}_{\ell}$ for every $q \in \overline{\mathcal{V}}$.

$>$ From now on we shall work in the Banach space $C^{r}\left(\overline{\mathcal{U}}_{\ell}\right)$. Applying the implicit function again, this time in $C^{r}\left(\overline{\mathcal{U}}_{\ell}\right)$, we find that there is some $\varepsilon_{\ell}>0$ such that whenever $\left\|g_{\ell}-f_{\ell}\right\|_{C^{r}\left(\overline{\mathcal{U}}_{\ell}\right)}<\varepsilon_{\ell}$, and $q=(p, w) \in \overline{\mathcal{V}}$, then the solution $\left(a_{-\ell}, b_{\ell}, \lambda_{\ell}\right)$ of the system:

$$
\begin{aligned}
a_{\ell}^{\ell} & =0 \\
D g_{\ell}\left(a_{-\ell}, b_{\ell}\right) & =\lambda_{\ell} q \\
g_{\ell}\left(a_{-\ell}, b_{\ell}\right) & =1
\end{aligned}
$$

has the property that $\left(a_{-\ell}, b_{\ell}\right) \in \mathcal{U}_{\ell}$, and depends smoothly on $g_{\ell}$ (in the $C^{r}\left(\overline{\mathcal{U}}_{\ell}\right)$ norm) and $q$. Set:

$$
\begin{aligned}
B\left(f_{\ell}, \varepsilon\right) & =\left\{g_{\ell} \in C^{r}\left(\overline{\mathcal{U}}_{\ell}\right) \mid\left\|g_{\ell}-f_{\ell}\right\|_{C^{r}\left(\overline{\mathcal{U}}_{\ell}\right)}<\varepsilon\right\} \\
B(f, \varepsilon) & =\prod_{\ell=1}^{L} B\left(f_{\ell}, \varepsilon\right)
\end{aligned}
$$

For fixed $q$, we can find the derivative with respect to $g_{\ell}$ as follows. Linearizing the system at $\left(a_{-\ell}, b_{\ell}, \lambda_{\ell}\right)$, and denoting by $\left(\alpha_{-\ell}, \beta_{\ell}, \mu_{\ell}\right)$ a tangent vector at that point, we relate them to the tangent vector $h_{\ell}$ at $g_{\ell}$ by:

$$
\begin{aligned}
{\left[D^{2} f_{\ell}\left(a_{-\ell}, b_{\ell}\right)\right]\left(\alpha_{-\ell}, \beta_{\ell}\right)-\mu_{\ell}(p, w) } & =-D h^{\ell}\left(a_{-\ell}, b_{\ell}\right) \\
{\left[D f_{\ell}\left(a_{-\ell}, b_{\ell}\right)\right]^{\prime}\left(\alpha_{-\ell}, \beta_{\ell}\right) } & =-h^{\ell}\left(a_{-\ell}, b_{\ell}\right)
\end{aligned}
$$

where $h^{\ell} \in C^{r}\left(\overline{\mathcal{U}}_{\ell}\right)$, and $\left(\alpha_{-\ell}, \beta_{\ell}, \lambda_{\ell}\right) \in R^{J} \times R$. For any given $h^{\ell}$, this is a system of $(L+K)$ equations with $(L+K)$ unknowns which is always uniquely solvable by Condition 3. By the implicit function theorem, the map $g_{\ell} \rightarrow\left(a_{-\ell}, b_{\ell}\right)$ from $B\left(f_{\ell}, \varepsilon_{\ell}\right)$ to $R^{J}$ is $C^{r-1}$ and its derivative is onto.

We now go back to $\varphi_{f}$. Recall that, for $w \in W_{1}$ and $g \in \mathcal{F}^{r}$, we define $\varphi_{g}^{1}(w)$, henceforth denoted by $\varphi_{f}(g, w)$, as the unique solution $p$ of the equation:

$$
p^{\prime}=w^{\prime} B_{g}(p, w)\left(I-A_{g}(p, w)\right)^{-1}
$$

so that $\varphi_{f}$ maps $B(f, \varepsilon) \times C$ into $R_{++}^{L}$. Using the implicit function theorem again, we see that $\varphi_{f}$ is $C^{r-1}$. We shall henceforth assume that $\varepsilon$ has been chosen so small 
that, for every $g \in B(f, \varepsilon)$, we have $G_{g} \subset \mathcal{V}$, where $G_{g}$ is the graph of $\varphi_{g}^{1}$ over $C$. By the envelope theorem, the derivative $D \varphi_{f}(f, w):(h, \omega) \rightarrow \pi$ sending tangent vectors $(h, \omega)$ at $(f, w)$ into tangent vectors $\pi$ to $p$ is given by:

$$
\pi^{\prime}=\omega^{\prime} B(I-A)^{-1}+\omega^{\prime}\left[\left(D_{f} B\right) h\right](I-A)^{-1}-\omega^{\prime} B(I-A)^{-1}\left[\left(D_{f} A\right) h\right](I-A)^{-1}
$$

where $\left(D_{f} A, D_{f} B\right)$ denotes the derivative at $g=f$ of the map

$$
g \rightarrow\left(A_{g}\left(\varphi_{f}(w), w\right), B_{g}\left(\varphi_{f}(w), w\right)\right)
$$

which is defined by relations (34) and (35) and has been shown to be onto.

We claim that $D \varphi_{f}(f, w)$ is onto for every $(f, w)$. Indeed, take any $\pi \in R^{L}$ and any $\omega \neq 0$. Pick some $X \in \mathcal{L}\left(R^{L}, R^{K}\right)$ such that $\pi^{\prime}-\omega^{\prime} B(I-A)^{-1}=\omega^{\prime} X(I-A)^{-1}$. Since $\left(D_{f} A, D_{f} B\right)$ is onto, we can find some $h$ such that $\left(D_{f} A\right) h=0$ and $\left(D_{f} B\right) h=$ $X$. Plugging this into (36) proves the claim.

Now go back to formula (33) defining $\Psi$. Set:

$$
\Delta(f, \varepsilon, C)=\left\{\left(g, w^{1}, w^{2}, \lambda\right) \in B(f, \varepsilon) \times C \times C \times R_{++} \mid w^{1} \neq w^{2}\right\}
$$

and denote by $\bar{\Psi}$ the restriction of $\Psi$ to $D(f, \varepsilon, C)$. Since $w^{1} \neq w^{2}$ in $W_{1}$, then $q^{1}=\left(\varphi_{f}\left(w^{1}\right), w^{1}\right)$ and $q^{2}=\left(\varphi_{f}\left(w^{2}\right), w^{2}\right)$ cannot be collinear. Plugging $q^{1}$ and $q^{2}$ in problem (5), we find different, solutions. This means that the corresponding $\left(a_{-\ell}\left(q^{1}\right), b_{\ell}\left(q^{1}\right)\right)$ and $\left(a_{-\ell}\left(q^{2}\right), b_{\ell}\left(q^{2}\right)\right)$ are distinct, so that the vectors $h\left(a_{-\ell}\left(q^{1}\right), b_{\ell}\left(q^{1}\right)\right)$ and $h\left(a_{-\ell}\left(q^{2}\right), b_{\ell}\left(q^{2}\right)\right)$ can be chosen independently. It then follows from the preceding argument that $D \bar{\Psi}$ is onto at every point. In particular, it has to be onto at every point where $\bar{\Psi}=0$, which means that $\bar{\Psi}$ is transversal to the origin.

By Thom's transversality theorem, generically with respect to $g \in B(f, \varepsilon)$, the partial map

$$
\bar{\Psi}_{g}:\left(w^{1}, w^{2}, \lambda\right) \rightarrow \bar{\Psi}\left(g, w^{1}, w^{2}, \lambda\right)
$$

from $\Delta^{C}$ to $R^{L}$ is transversal to the origin. Note that $g \rightarrow \bar{\Psi}_{g}$ is just the restriction of $g \rightarrow \Psi_{g}^{C}$ to $B(f, \varepsilon)$. It follows that every point $f \in \mathcal{F}^{r}$ has a neigbourhood $B^{r}(f, \varepsilon)$ where, generically with respect to $g \in B^{r}(f, \varepsilon)$, the map $\Psi_{g}^{C}$ is transversal to the origin.

We now derive Proposition ?? by applying another lemma. Recall first that a Lindelöf space is a topological space such that every covering by open subspaces has a countable subcover. Separable metric spaces (i.e. metric spaces which contain a countable dense subset) are Lindelöf, and every open subset of a Lindelöf space is Lindelöf; in particular, $\mathcal{F}^{r}$ is Lindelöf.

Lemma 39. Suppose the space $\Theta$ is Baire and Lindelöf and there is a property $P(\theta)$ such that every $\theta \in \Theta$ has an open neighbourhood $\mathcal{V}(\theta)$ on which $P(\theta)$ is generic. Then $P(\theta)$ is generic on $\Theta$. 
Proof. By the Lindelöf property, take a countable subcover $\mathcal{V}_{n}, n \in N$. Let $A_{n}$ be the set of points $\theta \in \mathcal{V}_{n}$ where $P(\theta)$ is true, and denote by $B_{n}$ the complement of $\overline{\left(\mathcal{V}_{n}\right)}$, the closure of $\mathcal{V}_{n}$, in $\Theta$. Set $C=\cap_{n}\left(A_{n} \cup B_{n}\right)$. By assumption, we have $A_{n} \supset \cap_{k} A_{n, k}$, where the $A_{n, k}, k \in N$, are open and dense in $\mathcal{V}_{n}$. Set $B_{n, k}=A_{n, k} \cup B_{n}$, clearly an open and dense subset in $\Theta$, and $C$ contains the intersection $\cap_{n, k} B_{n, k}$. Since the $\mathcal{V}_{n}, n \in N$, cover $\Theta$, every point $\theta \in C$ must belong to some $\mathcal{V}_{n}$, and therefore cannot belong to its complement $B_{n}$. It follows that it belongs to $A_{n}$, so that $P(\theta)$ is true on $C$, as announced.

Combining Lemmas ?? and ??, we find that, generically in $\mathcal{F}^{r}$, the partial map $\Psi_{f}^{C}: D^{C} \rightarrow R^{L}$ will be transversal to the origin in $R^{L}$. Taking a family of compact subsets $C_{n} \subset W_{1}$, such that $C_{n} \subset C_{n+1}$ and $\cup C_{n}=W_{1}$, and denote by $P_{n}(f)$ the property:

$$
P_{n}(f)=\left\{\Psi_{f}^{C_{n}} \text { is transversal to } 0 \in R^{L} \cdot\right\}
$$

Since $P_{n}(f)$ is generic in $\mathcal{F}^{r}$, so is $\wedge_{n} P_{n}(f)$. This means that $\Psi_{f}$ is transversal to the origin, and Proposition ?? is proved. So the set $\left(\Psi_{f}\right)^{-1}(0)$ either is a submanifold of codimension $L$ in $D$ (and hence of dimension $2 K-1-L$ ) or is empty. The latter is always the case when $2 K-1-L<0$, and Theorem?? follows.

If $w^{1} \neq w^{2} \in R_{++}^{K}$ are such that $\varphi\left(w^{1}\right)=\varphi\left(w^{2}\right)$, since $\varphi$ is positively homogeneous of degree one, we can find some positive $\lambda_{1}$ and $\lambda_{2}$ such that $w^{1} / \lambda_{1} \in W_{1}$ and $w^{2} / \lambda_{2} \in W_{1}$, so that:

$$
\frac{\lambda_{1}}{\lambda_{2}} \varphi\left(w^{1} / \lambda_{1}\right)=\varphi\left(w^{2} / \lambda_{2}\right)
$$

yielding a point $\left(w^{1} / \lambda_{1}, w^{2} / \lambda_{2}, \lambda_{1} / \lambda_{2}\right) \in\left(\Psi_{f}\right)^{-1}(0)$. In other words, the cone

$$
\left\{\left(w^{1}, w^{2}\right) \in R^{2 K} \mid w^{1} \neq w^{2}, \varphi\left(w^{1}\right)=\varphi\left(w^{2}\right)\right\}
$$

is generated by $\left(\Psi_{f}\right)^{-1}(0)$. If $\Psi_{f}$ is transversal to the origin, this cone is a submanifold with one more dimension than $\left(\Psi_{f}\right)^{-1}(0)$, namely $2 K-L$, and Theorem ?? follows. 